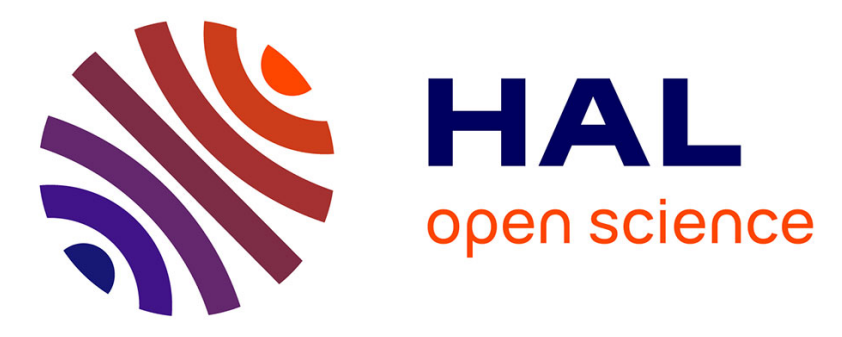

\title{
Pratiques alimentaires au Néolithique moyen : nouvelles données sur le site de Pontcharaud 2 (Puy-de-Dôme, Auvergne, France)
}

Gwenaëlle Goude, Aurore Schmitt, Estelle Herrscher, Gilles Loison, Sandrine Cabut, Guy André

\section{To cite this version:}

Gwenaëlle Goude, Aurore Schmitt, Estelle Herrscher, Gilles Loison, Sandrine Cabut, et al.. Pratiques alimentaires au Néolithique moyen: nouvelles données sur le site de Pontcharaud 2 (Puy-deDôme, Auvergne, France). Bulletin de la Société préhistorique française, 2013, 110 (2), pp.299-317. 10.3406/bspf.2013.14262 . hal-01411030

\author{
HAL Id: hal-01411030 \\ https://hal.science/hal-01411030
}

Submitted on 8 Dec 2016

HAL is a multi-disciplinary open access archive for the deposit and dissemination of scientific research documents, whether they are published or not. The documents may come from teaching and research institutions in France or abroad, or from public or private research centers.
L'archive ouverte pluridisciplinaire HAL, est destinée au dépôt et à la diffusion de documents scientifiques de niveau recherche, publiés ou non, émanant des établissements d'enseignement et de recherche français ou étrangers, des laboratoires publics ou privés. 


\title{
Pratiques alimentaires au Néolithique moyen : nouvelles données sur le site de Pontcharaud 2 (Puy-de-Dôme, Auvergne, France)
}

\author{
Gwenaëlle Goude, Aurore Schmitt, Estelle Herrscher, \\ Gilles LoIson, Sandrine CABut et Guy André
}

Résumé : Cet article présente de nouveaux résultats isotopiques $\left(\delta^{13} \mathrm{C}\right.$ and $\left.\delta^{15} \mathrm{~N}\right)$ obtenus sur un groupe humain du Néolithique moyen issu de l'ensemble funéraire du site de Pontcharaud 2 (Puy-de-Dôme, Auvergne, France). L'intérêt s'est initialement porté sur cette région notamment en raison de l'intéressante diversité écologique qu'offre cet espace géographique pour l'exploitation des ressources naturelles. L'acquisition de nouvelles données sur les comportements alimentaires au Néolithique dans une zone géographique, distincte de celles étudiées précédemment dans d'autres programmes de recherche, permet de développer une approche comparative relative aux choix alimentaires opérés par les Néolithiques en intégrant des aspects sociaux et environnementaux. Notre étude porte, d'une part, sur une analyse paléoalimentaire de la série ostéologique de Pontcharaud 2, par une approche isotopique intra-populationnelle intégrant les données biologiques et archéologiques et, d'autre part, sur une analyse inter-régionale entre trois zones géographiques : l'Auvergne, la Garonne et le Languedoc. L'objectif de cette comparaison est multiple et s'attache à savoir s'il existe des différences alimentaires entre les groupes méridionaux et plus septentrionaux de la deuxième moitié du $V^{e}$ millénaire, et si ces différences peuvent être mises en relation avec l'environnement propre de chacune de ces aires géographiques ou avec des choix culturels.

Le site de Pontcharaud 2 à Clermont-Ferrand en Auvergne a livré un vaste ensemble funéraire dont les premières datations radiométriques réalisées sur os proposent une fourchette chronologique de 4460-4030 BC cal. (LY7916 : 5640 \pm 70 BP; LY7918 : $5330 \pm 55$ BP; LY $7917: 5430 \pm 50$ BP) correspondant au Néolithique moyen 1 de la Limagne. Dans notre étude, deux nouvelles datations ont été réalisées sur des individus de l'inhumation multiple. Les résultats (5350 \pm 40 BP Poz-41914 : 4324-4051 BC cal.; 5380 土 40 BP Poz-41915 : 4335-4059 BC cal.) montrent que cet ensemble particulier a fonctionné dans le même intervalle chronologique que les sépultures. Une étude anthropologique a été réalisée préalablement aux analyses isotopiques. Selon le stade de croissance, différentes méthodes ont été appliquées pour estimer l'âge au décès des individus. Dans le traitement statistique, les individus immatures ont été répartis en 3 catégories : [0-4], [5-14] et [15-19] ans. Ce classement est défini par des critères biologiques. Le sexe des individus adultes a été déterminé dans un premier temps par une diagnose sur l'os coxal. La méthode de diagnose sexuelle probabiliste (DSP), élaborée à partir de données métriques, a été privilégiée. Les critères archéologiques retenus pour cette étude concernent, d'une part, la présence d'architecture pérenne ou non et, d'autre part, la distinction des sépultures individuelles des inhumations. Les isotopes stables $\left(\delta^{13} \mathrm{C}\right.$ and $\left.\delta^{15} \mathrm{~N}\right)$ du collagène osseux humain et animal ont été dosés afin d'appréhender l'origine environnementale des protéines consommées et le niveau trophique des individus. Au total, 37 adultes, 21 immatures et 7 animaux associés ont été échantillonnés et analysés par EA-IRMS. Les résultats isotopiques indiquent pour l'ensemble du groupe une importante consommation de protéines animales. La variabilité isotopique intra-groupe souligne des différences dans la proportion de protéines consommées entre les sujets ainsi que la possible consommation de ressources d'eau douce pour certains. La distribution isotopique ne semble pas liée à l'âge ou au sexe mais pourrait refléter des différences sociales et/ou géographiques, en relation avec le type d'inhumation. Une comparaison avec d'autres sites du Néolithique moyen en Languedoc et Garonne permet de proposer l'hypothèse d'une tendance régionale dans l'expression des modes de subsistance : d'une part, des groupes humains vivant à l'intérieur des terres, dans des zones plus ou moins vallonnées, probablement plus mobiles avec une économie pastorale développée et une consommation potentielle de ressources sauvages (aquatiques); d'autre part, des groupes humains vivant plus près des côtes, en plaine, probablement plus sédentaires, avec une économie de subsistance principalement tournée vers l'agriculture.

Mots clés : Néolithique moyen, Auvergne, alimentation, isotopes stables, carbone, azote, comparaison régionale.

Abstract: This paper presents new stable isotope data $\left(\delta^{13} \mathrm{C}\right.$ and $\left.\delta^{15} \mathrm{~N}\right)$ on the Middle Neolithic human group from Pontcharaud (Puy-de-Dôme, Auvergne, France), in order to determine a dietary pattern for both individuals and the entire group. The region considered is particularly interesting for its environmental diversity and for the possibility of exploiting various food resources. The acquisition of new Neolithic palaeodietary data in a geographic area not studied until 
now, and different from previous research, allows the development of a comparative approach to understanding foodstuff choices including social and environmental aspects. Indeed, data are analyzed within the environmental and social context, which helps to establish a regional comparison with other contemporary populations in the south of France, characterized by various subsistence economies. This comparison aims at understanding possible dietary differences between southern human groups and populations from central France during the second half of the 5th millennium BC, and checking if diet could be linked to a specific environment and/or to cultural choices.

The site of Pontcharaud 2 is located in Clermont Ferrand in Central France (Auvergne). It is composed of a large funerary complex from which radiocarbon dates from bones indicate a chronological range between 4460 and $4030 \mathrm{BC}$ cal. (LY7916: $5640 \pm 70$ BP; LY7918: $5330 \pm 55$ BP; LY 7917: $5430 \pm$ 50). In our study, two other radiocarbon dates were performed on human bones from the multiple deposit. The results (5350 $\pm 40 \mathrm{BP}$ Poz-41914: 4324-4051 BC cal.; 5380 \pm 40 BP Poz-41915: 4335-4059 BC cal.) also indicate that this specific group belongs to the same period as the rest of the necropolis. The number of burials and the preservation of human remains allowed much information to be obtained regarding bioarchaeological aspects as well as funerary practices. Indeed, osteological work was carried out prior to the isotopic analysis. According to the growth stage, different methods were used to estimate individuals' age at death. Immature subjects were divided into three subgroups in order to apply statistical processing: [0-4], [5-14] and [1519] years old. These subgroups were defined according to biological criteria. Sex assessment was performed on adult remains thanks to the 'DSP' method (probabilistic sex diagnosis) established on the basis of metric data. Archaeological criteria considered for this study are, on the one hand, the presence or absence of perennial funerary architecture, and, on the other, the distinction between individual or multiple burials. $\delta^{13} \mathrm{C}$ and $\delta^{15} \mathrm{~N}$ were analyzed on bone collagen from humans and animals in order to obtain individual dietary data, more particularly on the environments exploited (e. g. marine vs. terrestrial), and on the position of the individual within the trophic system (e. g. herbivore vs. carnivore). Adult $(\mathrm{n}=37)$ and immature $(\mathrm{n}=21)$ bones were selected with associated faunal material such as sheep/goat, cattle and pig $(\mathrm{n}=7)$, and the collagen extracted was measured by EA-IRMS. Results are consistent with the consumption of terrestrial animal protein for most of the individuals. The major part of the group seems to have consumed a significant quantity of herbivore meat coming from local resources. However, the wide nitrogen isotope range indicates different access to animal protein according to individuals. Thus, we suspect several subjects consumed ${ }^{15} \mathrm{~N}$ enriched resources. Among ${ }^{15} \mathrm{~N}$ enriched resources, and considering the local environment (semi-mountainous with an accessible fluvial network), freshwater fish and/or young animals could be good candidates. Isotopic variations seem not to be linked to sex or age, but could reflect social variations in relationship with burial practice specificities (individual burial vs. multiple burial or deposit). Indeed, statistical tests applied to compare individuals buried in single or double graves and individuals from the multiple deposit highlight a significant isotopic difference for both carbon and nitrogen. The seven subjects from this specific deposit seem to have had less animal protein intake than the other members of this community, and/or they had consumed food resources from a distinct isotopic environment. Seven individuals were excavated from this deposit: five adult men and two children. The status of these subjects comparatively to the rest of the group was widely debated and still commented. Our results underline the particularity of these people and suggest a link between their diet and their social status and/or their exogenous geographic origin.

A comparison with other Middle Neolithic sites from the southern region highlights various dietary patterns according to the region and the environment. The human group from Pontcharaud 2 shows the highest $\delta^{15} \mathrm{~N}$ values compared to groups located inland (Garonne), and those located close to the Mediterranean coast (Languedoc). To explain these differences, we propose the hypothesis of more animal protein intake and/or more ${ }^{15} \mathrm{~N}$ enriched protein intake (freshwater fish, young animals such as calf) for the Pontcharaud human group. This dietary pattern could be linked to local particularities such as climate and the semi-mountainous landscapes. Unfortunately no zooarchaeological study which could support our results is available for the moment. However, based on stable isotope analysis we can suggest regional trends in subsistence economy patterns: on the one hand, human groups living inland, in a hilly/semi-mountainous landscape, more mobile and consuming more wild resources (aquatic/terrestrial), considered as herders, and, on the other hand, a population located more on the plain, more sedentary, consuming no/few wild resources, considered as farmers.

Keywords: Middle Neolithic, Auvergne, Diet, Stable Isotopes, Carbon, Nitrogen, Regional comparison

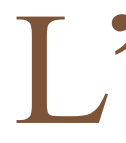

UTILISATION des marqueurs isotopiques pour la reconstitution des comportements alimentaires et des relations entre l'homme et son environnement est désormais bien établie dans le domaine de l'archéologie. Son application aux populations humaines du Néolithique depuis les premiers travaux de Tauber au Danemark (Tauber, 1981) s'est largement développée dans d'autres régions d'Europe, notamment pour étudier la problématique des changements alimentaires lors de la transition Mésolithique/Néolithique (e. g. Lubell et al., 1994; Bonsall et al., 1997; Lillie et Richards, 2000; Richards, 2003; Lidén et al., 2004;
Milner et al., 2004). En France, plusieurs sites ont fait l'objet de telles études au cours de ces dernières années (e. g. Bocherens et al., 2005 ; Herrscher et al., 2012), plus particulièrement dans le sud de la France, sous l'impulsion d'une action collective de recherche ${ }^{1}$. Dans cette région, des études comparatives, combinant à la fois des données anthropologiques (âge, sexe, stature, pathologie), archéologiques (e. g. pratiques funéraires), environnementales (faune, flore) et isotopiques $\left(\delta^{13} \mathrm{C}\right.$ et $\left.\delta^{15} \mathrm{~N}\right)$ ont été menées sur des sites datés du Néolithique moyen (ca 4500-3500 BC cal.; fig. 1; Goude, 2007; Le BrasGoude, 2008; Herrscher et Le Bras-Goude, 2008 et 2010; 


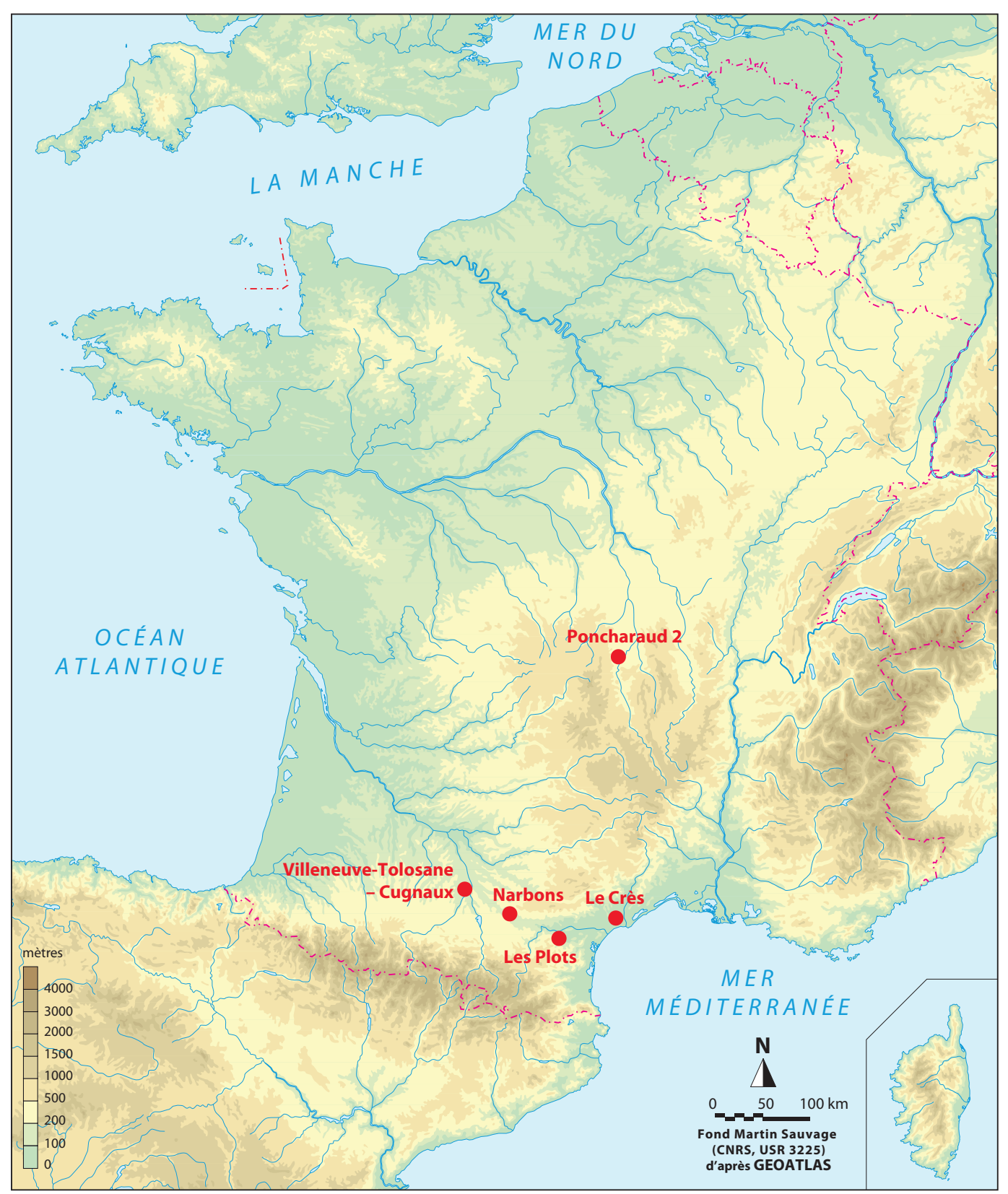

Fig. 1 - Localisation du site de Pontcharaud 2 et des sites de comparaison retenus.

Fig. 1 - Location of the Pontcharaud 2 site and other sites presented in this study.

Le Bras-Goude et Claustre, 2009; Le Bras-Goude et al. 2009, 2012). L'intérêt s'est initialement porté sur cette région, d'une part, en raison du nombre de sites ayant livré des restes humains, et, d'autre part, en raison de l'intéressante diversité écologique qu'offre cet espace géographique pour l'exploitation des ressources naturelles (milieu marin, milieu terrestre, plaines, zones vallonnées). Plus récemment, un nouveau programme de recherche $^{2}$ a permis de poursuivre ces analyses sur le site néolithique moyen de Pontcharaud 2 en Auvergne (fig. 1). L'acquisition de nouvelles données sur les comportements alimentaires au Néolithique dans une région distincte de celles étudiées précédemment permet de développer une approche comparative relative aux choix alimentaires opérés par les néolithiques en considérant/ intégrant des aspects sociaux et environnementaux. Notre étude porte donc, d'une part, sur une analyse paléoalimentaire de la série ostéologique de Pontcharaud 2, par une approche isotopique intra-populationnelle intégrant les données biologiques et archéologiques et, d'autre part, sur une analyse inter-régionale entre trois zones géographiques : l'Auvergne, la Garonne et le Languedoc. L'objectif de cette comparaison est multiple : 1) existe-t-il des différences alimentaires entre les groupes méridionaux et plus septentrionaux de la deuxième moitié du $\mathrm{V}^{\mathrm{e}}$ millénaire? 2) Peuvent-elles être mises en relation avec l'environnement propre de chacune de ces aires géographiques ou avec des choix culturels? 


\section{PRÉSENTATION DE LA SÉRIE, DU CONTEXTE ARCHÉOLOGIQUE ET ENVIRONNEMENTAL}

e site de Pontcharaud 2 à Clermont-Ferrand en

Auvergne a livré un vaste ensemble funéraire composé de 58 structures et 71 individus (Civetta et al., 2009). L'opération archéologique a été réalisée par l'AFAN en 1986. Les premières datations radiométriques réalisées sur os proposent une fourchette chronologique de 4460-4030 BC cal. (LY7916 : $5640 \pm 70$ BP; LY7918 : $5330 \pm 55$ BP; LY $7917: 5430 \pm 50$ BP) correspondant au Néolithique moyen 1 de la Limagne (Loison, 1998). Plus récemment deux nouvelles datations ont été réalisées sur les individus 31 et 36 (sur le collagène extrait pour cette étude) issus de l'ensemble multiple 31-37 sur lequel nous reviendrons. Les résultats $(5350 \pm 40 \mathrm{BP}$ Poz41914 : 4324-4051 BC cal.; $5380 \pm 40$ BP Poz-41915 : 4335-4059 BC cal.) montrent que cette inhumation particulière a fonctionné dans le même intervalle chronologique que les autres. La majorité des défunts a bénéficié d'une sépulture individuelle. Toutefois, quatre sépultures présentent des dépôts simultanés (26-26b, 29-30, 52-53, 31-37; Loison, 1998). Deux tombes présentent des éléments d'architecture pérenne (12 et 39).

La série a fait l'objet de plusieurs études anthropologiques. L'état sanitaire a été étudié par Billard (2010). Les infections et les carences protéino-énergétiques (anémie) sont rares. Les conditions de vie étaient probablement plus défavorables pour les femmes durant l'enfance. La série de Pontcharaud 2 présente une forte fréquence des indicateurs de stress biomécaniques d'activité liées à des contraintes résultant d'efforts physiques intenses et répétés impliquant le rachis lombaire $(e . g$. port de charges lourdes). Ce sont surtout les jeunes adultes qui sont touchés par ces lésions osseuses sans distinction de sexe. Ils sont d'ailleurs inhumés dans le quart nord-ouest de l'ensemble funéraire. Une étude biométrique a révélé un dimorphisme sexuel marqué de la forme et du format des os longs (Civetta et al., 2009). Ces différences peuvent être liées à une activité physique différenciée selon le sexe. La forme crânienne des femmes étant plus hétérogène que celle des hommes, l'hypothèse d'une mobilité accrue des femmes a été suggérée (Civetta et al., 2009).

L'environnement au Néolithique en Auvergne est notamment caractérisé par un couvert forestier riche. L'étude des données paléoenvironnementales a révélé un fort impact anthropique en raison de la mobilité des groupes humains, du défrichement aux alentours des points d'eau pour le bétail et du développement de la céréaliculture sur les versants, suivant une organisation de complémentarité entre les zones palustres et les zones plus montagneuses (Trément et al., 2006). Aucune étude archéozoologique ni botanique n'a été publiée pour le moment. Les seules informations fauniques concernent le niveau épicardial du site où l'on retrouve un grand nombre de restes de faune sauvage, comme l'aurochs (comm. pers. V. Forest). Par contre, de plus amples études archéozoologiques menées sur des aires géographiques limitrophes (Bréhard, 2011; Bréhard et al., 2010) permettent d'avoir une vision plus précise de l'économie pastorale du Néolithique moyen dans la moitié sud de la France (vallée du Rhône, Languedoc et Garonne). Les travaux réalisés par Bréhard et al. (2010) et Bréhard (2011) soulignent notamment l'importance des bovins dans le cheptel domestique, mais insistent également sur la structuration et la complémentarité entre les sites d'abri, destinés aux troupeaux d'ovicaprins et les sites de plein air, plus orientés pour l'exploitation des bovins dans le but d'une production mixte (lait + viande).

\section{MÉTHODE ET MATÉRIEL}

T a méthode utilisée pour répondre aux questions relatives aux modes de subsistance est l'analyse des isotopes stables (carbone et azote) contenus dans le collagène de l'os, protéine synthétisée à partir des acides aminés issus de l'alimentation. Ils sont des marqueurs des tendances des régimes alimentaires et leur analyse permet une approche anthropologique directe et pertinente des comportements humains, à l'échelle individuelle et populationnelle. Le dosage des isotopes stables du carbone et de l'azote $\left(\delta^{13} \mathrm{C}\right.$ et $\left.\delta^{15} \mathrm{~N}\right)$ du collagène osseux permet de connaître dans quel milieu (e.g. marin, terrestre, tempéré) l'individu a puisé ses ressources ainsi que sa place dans la chaîne alimentaire. En effet, les végétaux à la base de la chaîne alimentaire ont des valeurs isotopiques $\left(\delta^{13} \mathrm{C}\right.$ et $\left.\delta^{15} \mathrm{~N}\right)$ qui varient en fonction du type de photosynthèse $\left(\mathrm{C}_{3}, \mathrm{C}_{4}\right)$, de l'environnement (aride, tempéré, marin, terrestre, de plaine ou de sous-bois) et des espèces (végétaux fixateurs d'azote telles que les légumineuses ou non fixateurs d'azote; Park et Epstein, 1960). Ces différences isotopiques se répercutent tout au long de la chaîne alimentaire avec un enrichissement en isotope lourd $\left({ }^{13} \mathrm{C}\right.$ et ${ }^{15} \mathrm{~N}$ ) à chaque maillon du réseau trophique (DeNiro et Epstein, 1978). Les différences de $\delta^{13} \mathrm{C}$ et $\delta^{15} \mathrm{~N}$ observées entre le collagène de deux individus de niveaux trophiques successifs (e.g. de l'herbivore au carnivore) sont généralement de $+0,0$ à $+1,0 \%$ pour le carbone et de $+3,0$ à $+5,0 \%$ pour l'azote (DeNiro et Epstein, 1978; DeNiro et Epstein 1981; Minagawa et Wada, 1984; Bocherens et Drucker, 2003). Les tissus osseux se renouvellent tout au long de la vie de l'individu avec une vitesse de turnover complet estimée à ca 10-15 ans (Ambrose, 1990). Cependant, cette cinétique dépend fortement de plusieurs facteurs tels que l'état sanitaire, les hormones et en particulier l'âge du sujet. En effet, des travaux menés sur l'actuel montrent que chez un enfant de 10 ans le renouvellement de l'os cortical est onze fois plus rapide que chez l'adulte et six fois plus rapide chez un jeune de 15 ans (Valentin, 2003). Par conséquent, plus le sujet est jeune, plus la signature isotopique enregistrée $\left(\delta^{13} \mathrm{C}\right.$ et $\left.\delta^{15} \mathrm{~N}\right)$ témoigne d'une alimentation récente. Des ossements animaux de différentes espèces (principalement moutons/chèvres et bœufs adultes) ont également fait l'objet d'une analyse 
afin de définir une référence isotopique pour des régimes alimentaires connus dans l'environnement local du site. Cette étape est essentielle pour interpréter au mieux les données obtenues sur les sujets humains (Bösl et al., 2006; Britton et al., 2008; Herrscher et Le Bras-Goude, 2010). Le collagène osseux a été extrait par la méthode Longin (1971), réadaptée par Bocherens et al. (1991) au laboratoire de biochimie de l'UMR 7269 « LAMPEA » (Aix-en-Provence). Les teneurs élémentaires et les mesures isotopiques ont été réalisées sur $0,5 \mathrm{mg}$ de collagène lyophilisé, par EA-IRMS (Europa Scientific 20-20, Iso-Analytical Ltd., Crewe, Royaume-Uni). L'erreur analytique, calculée à partir des différents standards, est de maximum $0,2 \%$ pour l'azote et de $0,05 \%$ o pour le carbone $^{3}$. Seuls les échantillons respectant l'ensemble des critères de conservation seront pris en compte pour l'interprétation des valeurs isotopiques. Les critères choisis sont ceux communément utilisés dans la littérature (Ambrose, 1990; DeNiro, 1985; van Klinken, 1999) : un rendement d'extraction $\geqslant 10 \mathrm{mg} / \mathrm{g}$, une concentration en $\mathrm{C}$ et $\mathrm{N}$ supérieure ou égale à $30 \%$ et $11 \%$ respectivement et un $\mathrm{C} / \mathrm{N}$ atomique compris entre 2,9 et 3,6. Pour résumer, cette méthode permet donc d'avoir des informations sur : 1) l'écosystème dans lequel les individus humains ont collecté leurs ressources alimentaires, 2) la place des individus dans la chaîne alimentaire et 3) les différences de comportements sociaux et économiques (Schoeninger et al., 1983; Schoeninger et Moore, 1992; Bocherens et Drucker, 2003).

Au total 37 adultes et 21 sujets immatures composent notre corpus d'étude, soit $80 \%$ de la série mise au jour (tableau 1). D'une manière générale, les corticales d'os longs ont préférentiellement été prélevées et plus particulièrement les fibulas (pour $55 \%$ des sujets).

Préalablement à ces échantillonnages, une étude anthropologique a été réalisée. Selon le stade de croissance, différentes méthodes ont été appliquées pour estimer l'âge au décès des individus. En règle générale, l'utilisation de la croissance osseuse pour estimer l'âge au décès des enfants est fortement déconseillée, mais lorsque le décès est précoce, les facteurs génétiques et environnementaux n'ont pas eu le temps d'influencer le développement biologique d'un individu (Scheuer et Black, 2000). Par conséquent, pour les enfants de moins d'un an, la stature a été estimée à partir des équations issues des régressions proposées par Sellier (1993 dans Schmitt et Georges, 2008) à partir de la longueur diaphysaire. Ensuite, l'âge a été estimé en appliquant les régressions logarithmiques d'Olivier et Pineau (1958) à partir des données sur la stature. Pour les individus de plus d'un an, plusieurs méthodes ont été appliquées. L'âge dentaire étant un indicateur plus performant que l'âge osseux, il a été privilégié. Pour l'estimation à partir des dents, nous avons utilisé le référentiel d'éruption et de minéralisation dentaire proposé par Ubelaker (1989). Lorsque les dents étaient absentes ou la minéralisation des dents permanentes complète, l'âge a été estimé à partir de la fusion des points d'ossification secondaire (Birkner, 1980). Dans le traitement statistique, les individus immatures ont été répartis en trois catégories : [0-4], [5-14] et [15-19] ans. Ce classement est défini par des critères biologiques. Les enfants en bas âge sont ceux décédés entre la naissance et 4 ans inclus. La limite supérieure correspond à l'âge maximal présumé de la fin de l'allaitement. Les enfants décédés entre 5 et 14 ans inclus constituent la deuxième classe. Les bornes de cette classe correspondent aux limites supérieures et inférieures des deux autres classes. Par convention, la classe des adolescents correspond à ceux décédés entre 15 et 19 ans (Bruzek et al., 2005). L'identification d'un adolescent prend en compte la maturation dentaire et le début de fusion des points d'ossification. Le sujet S22 n'a pas pu bénéficier d'un âge dentaire et donc d'un âge individuel, nous l'avons classé directement dans la classe [5-14] car le format de ses os longs est plus élevé que les sujets de la classe [0-4]. L'individu S25, bien qu'à cheval sur deux catégories, a été placé dans la classe [0-4] dans la mesure où l'intervalle chronologique de l'estimation le situe principalement dans cette catégorie.

Le sexe des individus adultes a été déterminé dans un premier par temps par une diagnose sur l'os coxal. La méthode de diagnose sexuelle probabiliste (DSP) élaborée à partir de données métriques a été privilégiée (Murail et al., 2005). Toutefois, pour certains sujets, le minimum de quatre mesures requis pour son application n'étant pas atteint, nous avons eu recours à la méthode visuelle élaborée par Bruzek (2002). Un sexe a pu être attribué à vingt-huit individus. L'os coxal étant absent ou trop mal conservé pour neuf adultes, nous avons réalisé une diagnose sexuelle secondaire en réalisant des analyses discriminantes sur les mesures des os longs des individus sexés de façon primaire (sur l'os coxal) que nous avons appliquées aux sujets non sexés. Cette étude a été réalisée sur la totalité des individus adultes de la série (SalibaSerre et Schmitt, 2012) et pas uniquement sur l'échantillon de la présente étude. Un sexe a été ainsi attribué à trois sujets supplémentaires. Six sujets demeurent de sexe indéterminé. Pour l'estimation de l'âge des adultes, nous avons appliqué les méthodes fondées respectivement sur la surface sacro-pelvienne iliaque et la symphyse pubienne (Schmitt, 2005, 2008). Les individus ont ensuite été répartis en trois catégories : [20-39], [40-59] et [plus de 60 ans]. L'âge des sujets S7 et S16 étant à cheval sur deux catégories, nous avons sélectionné une classe unique en tenant compte de l'état bucco-dentaire général de ces individus.

Les équations de régression appliquées à la longueur du fémur, de l'humérus et de la fibula définies par Trotter et Gleser et revues par Cleuvenot et Hoüet (Cleuvenot et Houët, 1993) ont permis d'estimer la stature. Le calcul de la stature des individus adultes a été possible pour trentecinq individus adultes.

Les critères archéologiques retenus pour cette étude concernent, d'une part, la présence d'architecture pérenne ou non : la structure 12 est un coffre composé de dalles de calcaire et la structure 39 est une fosse aménagée de dalles et de blocs calcaires. Les autres étaient probablement des inhumations en fosse (Loison, 1998). D'autre part, nous 


\begin{tabular}{|c|c|c|c|c|c|c|c|c|c|c|c|c|c|}
\hline Sépulture & $\mathrm{N}^{\circ}$ & $\mathrm{N}^{\circ}$ labo & $\begin{array}{l}\delta^{13} \mathrm{C} \\
(\% 0)\end{array}$ & $\begin{array}{l}\delta^{15} \mathrm{~N} \\
(\% 0)\end{array}$ & $\% \mathrm{C}$ & $\% \mathrm{~N}$ & $\mathrm{C} / \mathrm{N}$ & $\begin{array}{c}\text { Rdt } \\
(\mathrm{mg} / \mathrm{g})\end{array}$ & Sexe & $\begin{array}{l}\text { Classe } \\
\text { d'âge }\end{array}$ & $\begin{array}{c}\text { Stature } \\
(\mathrm{cm})\end{array}$ & $\begin{array}{l}\text { Archi- } \\
\text { tecture } \\
\text { pérenne }\end{array}$ & Type de tombe \\
\hline 3 & 3 & NFN01 & $-19,9$ & 10,0 & 40,3 & 14,6 & 3,2 & 89,5 & & $60+$ & & absente & individuelle \\
\hline 4 & 4 & NFN02 & $-20,3$ & 11,6 & 42,5 & 15,3 & 3,2 & 68,5 & & $40-59$ & 167,1 & absente & individuelle \\
\hline 5 & 5 & NFN03 & $-19,8$ & 10,3 & 35,1 & 12,7 & 3,2 & 23,2 & $\mathrm{M}$ & $20-39$ & 160,1 & absente & individuelle \\
\hline 7 & 7 & NFN05 & $-20,1$ & 10,6 & 32,7 & 11,8 & 3,2 & 28,7 & & $60+$ & 156,3 & absente & individuelle \\
\hline 8 & 8 & NFN06 & $-20,2$ & 10,6 & 30,6 & 10,8 & 3,3 & 26,4 & & & & absente & individuelle \\
\hline 9 & 9 & NFN07 & $-20,2$ & 9,4 & 41,3 & 14,6 & 3,3 & 21,2 & $\mathrm{~F}$ & & 161,5 & absente & individuelle \\
\hline 10 & 10 & NFN08 & $-19,7$ & 10,4 & 45,4 & 16,1 & 3,3 & 34,7 & M & & 156,4 & absente & individuelle \\
\hline 12 & 12 & NFN10 & $-20,3$ & 9,6 & 39,1 & 14,1 & 3,2 & 43,9 & $\mathrm{~F}$ & $20-39$ & 147,7 & présente & individuelle \\
\hline 13 & 13 & NFN11 & $-19,9$ & 10,5 & 41,0 & 14,4 & 3,3 & 18,3 & F & $20-39$ & 160,7 & absente & individuelle \\
\hline 14 & 14 & NFN12 & $-20,0$ & 10,2 & 37,5 & 13,4 & 3,2 & 55,8 & $\mathrm{~F}$ & & 153,4 & absente & individuelle \\
\hline 15 & 15 & NFN13 & $-19,9$ & 9,9 & 41,3 & 14,5 & 3,3 & 19,3 & M & $60+$ & 161,5 & absente & individuelle \\
\hline 16 & 16 & NFN14 & $-19,8$ & 10,4 & 38,5 & 13,9 & 3,2 & 46,7 & M & $60+$ & 162,6 & absente & individuelle \\
\hline 18 & 18 & NFN15 & $-19,8$ & 10,3 & 32,1 & 11,5 & 3,3 & 22,0 & M & $40-59$ & 159,5 & absente & individuelle \\
\hline 23 & 23 & NFN17 & $-20,4$ & 10,7 & 40,7 & 14,1 & 3,4 & 29,1 & $\mathrm{~F}$ & 20-39 & 152,1 & absente & individuelle \\
\hline $26-26 b$ & 26 & NFN18 & $-20,2$ & 11,8 & 38,8 & 13,9 & 3,2 & 60,1 & & $40-59$ & 149,7 & absente & multiple (2) \\
\hline 27 & 27 & NFN19 & $-19,8$ & 10,6 & 42,5 & 15,2 & 3,2 & 14,4 & M & $20-39$ & 163,1 & absente & individuelle \\
\hline \multirow[t]{2}{*}{$29-30$} & 29 & NFN21 & $-19,9$ & 10,0 & 44,1 & 15,7 & 3,3 & 25,3 & $\mathrm{~F}$ & $20-39$ & 157,2 & absente & multiple (2) \\
\hline & 30 & NFN22 & $-20,1$ & 8,7 & 40,8 & 15,7 & 3,0 & 38,7 & $\mathrm{~F}$ & $20-39$ & 148,4 & absente & multiple (2) \\
\hline \multirow[t]{5}{*}{$31-37$} & 31 & NFN23 & $-20,4$ & 9,4 & 47,4 & 16,8 & 3,3 & 30,6 & $\mathrm{M}$ & $40-59$ & 154,7 & absente & multiple (7) \\
\hline & 32 & NFN24 & $-20,9$ & 9,7 & 44,6 & 15,8 & 3,3 & 36,4 & M & $20-39$ & 155,8 & absente & multiple (7) \\
\hline & 33 & NFN25 & $-20,7$ & 9,4 & 43,7 & 15,5 & 3,3 & 25,9 & M & $20-39$ & 163,1 & absente & multiple (7) \\
\hline & 36 & NFN26 & $-20,5$ & 9,7 & 43,5 & 15,5 & 3,3 & 32,3 & M & $40-59$ & 167,2 & absente & multiple (7) \\
\hline & 37 & NFN27 & $-20,8$ & 9,3 & 42,2 & 15,1 & 3,2 & 24,1 & M & $40-59$ & 166,6 & absente & multiple (7) \\
\hline 38 & 38 & NFN28 & $-20,2$ & 9,8 & 46,2 & 16,6 & 3,2 & 89,5 & $\mathrm{~F}$ & $20-39$ & 146,7 & absente & individuelle \\
\hline 39 & 39 & NFN29 & $-20,4$ & 11,3 & 40,8 & 14,5 & 3,3 & 21,0 & $\mathrm{M}$ & $40-59$ & 158,4 & présente & individuelle \\
\hline 41 & 41 & NFN31 & $-19,8$ & 10,3 & 34,9 & 12,6 & 3,2 & 27,5 & $\mathrm{~F}$ & $40-59$ & 159,7 & absente & individuelle \\
\hline 45 & 45 & NFN33 & $-20,2$ & 10,5 & 43,7 & 15,5 & 3,3 & 40,0 & M & $40-59$ & 170,5 & absente & individuelle \\
\hline 51 & 51 & NFN37 & $-20,3$ & 9,8 & 43,8 & 15,6 & 3,3 & 40,0 & $\mathrm{~F}$ & $40-59$ & 149,1 & absente & individuelle \\
\hline \multirow{2}{*}{$52-53$} & 52 & NFN38 & $-20,7$ & 11,3 & 45,5 & 15,9 & 3,3 & 34,2 & $\mathrm{~F}$ & & 157,2 & absente & multiple (2) \\
\hline & 53 & NFN39 & $-20,3$ & 9,9 & 43,4 & 15,1 & 3,3 & 30,1 & $F$ & $20-39$ & 142,9 & absente & multiple (2) \\
\hline 55 & 55 & NFN40 & $-20,3$ & 9,7 & 47,6 & 17,3 & 3,2 & 77,9 & $\mathrm{~F}$ & $20-39$ & 143,8 & absente & individuelle \\
\hline 60 & 60 & NFN41 & $-20,1$ & 10,5 & 41,4 & 14,8 & 3,2 & 13,2 & M & $40-59$ & 159,5 & absente & individuelle \\
\hline 61 & 61 & NFN42 & $-20,4$ & 10,6 & 42,8 & 15,4 & 3,2 & 48,4 & $\mathrm{~F}$ & $60+$ & 142,1 & absente & individuelle \\
\hline 62 & 62 & NFN43 & $-20,7$ & 13,0 & 38,8 & 13,8 & 3,3 & 19,0 & $\mathrm{~F}$ & & 158,1 & absente & individuelle \\
\hline 63 & 63 & NFN44 & $-20,1$ & 10,1 & 42,0 & 14,1 & 3,5 & 36,3 & & $40-59$ & 155,2 & absente & individuelle \\
\hline 66 & 66 & NFN45 & $-19,9$ & 10,6 & 44,3 & 15,7 & 3,3 & 44,8 & M & $20-39$ & 162,4 & absente & individuelle \\
\hline 69 & 69 & NFN46 & $-20,2$ & 9,9 & 44,9 & 16,2 & 3,2 & 95,3 & F & $20-39$ & 155,8 & absente & individuelle \\
\hline 50 & 50 & NFN36 & $-20,1$ & 9,5 & 40,5 & 14,3 & 3,3 & 21,9 & & $15-19$ & & absente & individuelle \\
\hline 2 & 2 & NFN47 & $-19,4$ & 11,0 & 42,5 & 15,5 & 3,2 & 77,2 & & $0-4$ & & absente & individuelle \\
\hline 20 & 20 & NFN50 & $-19,9$ & 11,1 & 38,7 & 14,1 & 3,2 & 51,9 & & $0-4$ & & absente & individuelle \\
\hline 22 & 22 & NFN51 & $-20,5$ & 10,0 & 43,2 & 15,8 & 3,2 & 90,4 & & $5-14$ & & absente & individuelle \\
\hline 24 & 24 & NFN52 & $-19,8$ & 12,0 & 42,1 & 15,4 & 3,2 & 57,9 & & $0-4$ & & absente & individuelle \\
\hline 25 & 25 & NFN53 & $-19,7$ & 11,9 & 41,0 & 15,2 & 3,1 & 38,1 & & $0-4$ & & absente & individuelle \\
\hline $26-26$ bis & 26 bis & NFN54 & $-19,8$ & 10,3 & 41,3 & 15,1 & 3,2 & 60,0 & & $0-4$ & & absente & multiple (2) \\
\hline \multirow[t]{2}{*}{$31-37$} & 34 & NFN55 & $-20,9$ & 9,7 & 37,1 & 13,5 & 3,2 & 24,6 & & $5-14$ & & absente & multiple (7) \\
\hline & 35 & NFN56 & $-20,7$ & 9,9 & 44,5 & 16,2 & 3,2 & 44,0 & & $5-14$ & & absente & multiple (7) \\
\hline 42 & 42 & NFN57 & $-19,7$ & 9,5 & 30,1 & 11,1 & 3,1 & 31,6 & & $5-14$ & & absente & individuelle \\
\hline 43 & 43 & NFN58 & $-20,7$ & 11,4 & 36,0 & 13,0 & 3,2 & 37,4 & & $5-14$ & & absente & individuelle \\
\hline 46 & 46 & NFN59 & $-20,1$ & 10,4 & 36,3 & 13,2 & 3,2 & 62,6 & & $5-14$ & & absente & individuelle \\
\hline 54 & 54 & NFN60 & $-18,9$ & 12,7 & 39,4 & 14,3 & 3,2 & 49,7 & & $0-4$ & & absente & individuelle \\
\hline 56 bis & 56 bis & NFN62 & $-20,7$ & 10,9 & 45,3 & 16,6 & 3,2 & 60,7 & & $5-14$ & & absente & individuelle \\
\hline 57 & 57 & NFN63 & $-20,4$ & 11,0 & 33,6 & 12,3 & 3,2 & 20,3 & & $5-14$ & & absente & individuelle \\
\hline 58 & 58 & NFN64 & $-20,3$ & 10,3 & 45,6 & 16,7 & 3,2 & 50,6 & & $15-19$ & & absente & individuelle \\
\hline 59 & 59 & NFN65 & $-20,4$ & 9,4 & 47,6 & 17,6 & 3,1 & 51,3 & & $5-14$ & & absente & individuelle \\
\hline 64 & 64 & NFN66 & $-20,1$ & 10,0 & 31,0 & 11,7 & 3,1 & 29,8 & & $15-19$ & & absente & individuelle \\
\hline 65 & 65 & NFN67 & $-19,7$ & 12,1 & 34,6 & 12,5 & 3,2 & 31,7 & & $0-4$ & & absente & individuelle \\
\hline 67 & 67 & NFN68 & $-20,2$ & 8,6 & 44,4 & 16,7 & 3,1 & 47,2 & & $5-14$ & & absente & individuelle \\
\hline 68 & 68 & NFN69 & $-20,1$ & 9,8 & 40,1 & 14,8 & 3,1 & 58,4 & & $15-19$ & & absente & individuelle \\
\hline Bovin & & NFNA01 & $-22,4$ & 8,0 & 40,4 & 14,2 & 3,3 & 24,7 & & & & & \\
\hline Bovin & & NFNA07 & $-22,0$ & 7,0 & 42,6 & 15,1 & 3,3 & 28,3 & & & & & \\
\hline Ovicaprin & & NFNA02 & $-21,9$ & 6,1 & 42,9 & 15,2 & 3,3 & 28,2 & & & & & \\
\hline Ovicaprin & & NFNA03 & $-21,1$ & 5,4 & 36,1 & 12,7 & 3,3 & 21,3 & & & & & \\
\hline Ovicaprin & & NFNA08 & $-21,0$ & 6,0 & 42,1 & 15,0 & 3,3 & 21,8 & & & & & \\
\hline Ovicaprin & & NFNA05 & $-21,4$ & 5,7 & 41,3 & 14,6 & 3,3 & 23,1 & & & & & \\
\hline Suiné & & NFNA04 & $-20,7$ & 5,9 & 42,8 & 15,3 & 3,3 & 47,5 & & & & & \\
\hline
\end{tabular}

Tabl. 1 - Données isotopiques $\left(\delta^{13} \mathrm{C}, \delta^{15} \mathrm{~N}\right)$, élémentaires $(\% \mathrm{C}, \% \mathrm{~N}, \mathrm{C} / \mathrm{N})$, du rendement d'extraction (Rdt), des informations biologiques et archéologiques des sujets humains et animaux de Pontcharaud 2.

Table 1 - Presentation of stable isotope values, element contents $(\% \mathrm{C}, \% \mathrm{~N}), \mathrm{C} / \mathrm{N}$ ratios, extraction yield and biological and archaeological data linked to human and animal subjects studied at Pontcharaud 2. 
avons distingué les sépultures individuelles des « sépultures » ou inhumations multiples : trois sont doubles (29$30 ; 26-26 b ; 52-53)$ et la quatrième $(31-37)$ a livré sept sujets. En effet, le dépôt simultané de plusieurs sujets dans une même tombe témoigne d'un geste funéraire pouvant être lié à un aléa de la mortalité du groupe, notamment pour les sépultures doubles (Chambon et Leclerc, 2007). De plus, si un dépôt simultané excède trois sujets, la mortalité naturelle du groupe est discutable (ibid.).

Des statistiques descriptives ont été calculées pour l'ensemble des variables et en particulier pour les mesures isotopiques. La moyenne et l'écart-type étant très sensibles aux valeurs extrêmes, leur utilisation sur des échantillons de petite taille n'est pas recommandée. Nous leur avons donc préféré des indicateurs de tendance centrale et de dispersion plus robustes, à savoir la médiane $(\mathrm{Me})$ et l'intervalle interquartile (IIQ; intervalle délimité par les $1^{\text {er }}$ et $3^{\text {e }}$ quartiles). La moyenne et l'écart-type (SD) ont toutefois été calculés pour les échantillons de taille suffisante. Toujours en raison des faibles effectifs étudiés, le rho de Spearman a été utilisé comme mesure de corrélation. Des tests exacts de Wilcoxon ont été utilisés pour comparer les mesures isotopiques en carbone et en azote de deux groupes indépendants, des tests de KruskalWallis (approximation de Monte-Carlo, 10000 répétitions) dans le cas de plus de deux groupes. Ces tests non paramétriques et notamment leur version exacte ou approximée par la méthode de Monte-Carlo sont particulièrement indiqués dans le cas de très petits effectifs. De plus, pour remédier aux problèmes posés par la mul-

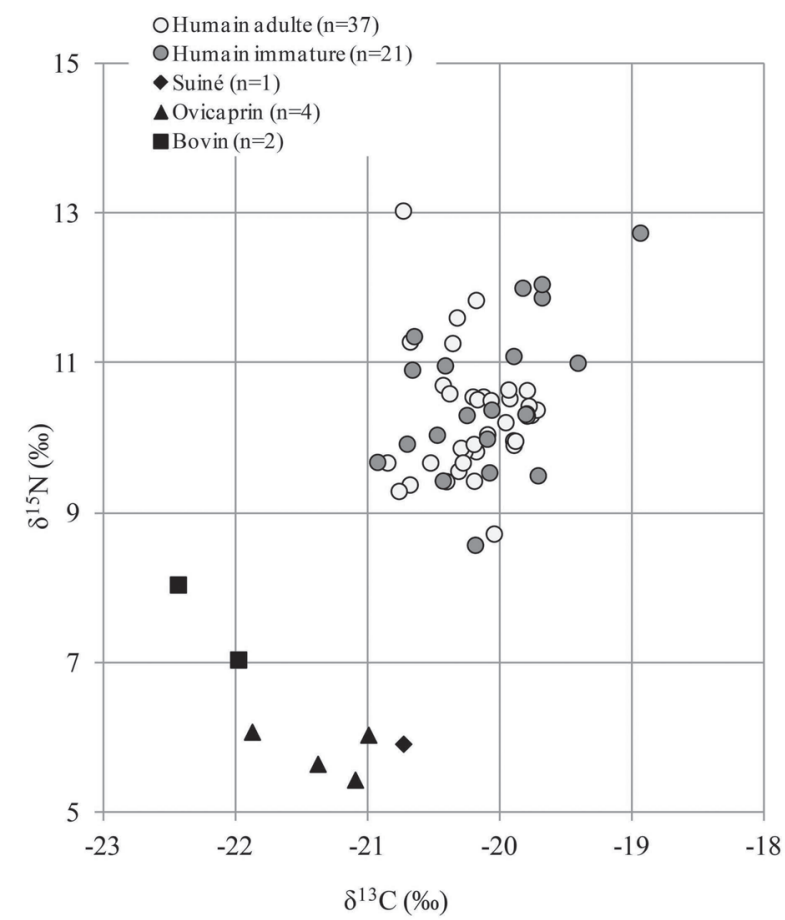

Fig. 2 - Distribution des valeurs isotopiques $\left(\delta^{13} \mathrm{C}, \delta^{15} \mathrm{~N}\right)$ des sujets humains et de la faune de Pontcharaud 2.

Fig. 2 - Stable isotope distribution of human and animal subjects from Pontcharaud 2. tiplicité des tests, nous avons appliqué la correction du False Discovery Rate (FDR; Benjamini et al., 1995) aux p-values des comparaisons deux à deux. Les analyses statistiques ont été réalisées avec le logiciel R v2.13.2 (R Development Core Team, 2011).

\section{L'ALIMENTATION PROTÉINIQUE DES SUJETS DE PONTCHARAUD 2}

$\mathrm{L}$ es différents critères de conservation spécifiés auparavant sont respectés pour tous les échantillons présentés dans le tableau 1. Les valeurs isotopiques de la faune s'étendent de $-22,4$ à $-20,7 \%$ o $(\mathrm{Me}=-21,4 \%$; IIQ $=[-22,0 ;-21,0])$ pour le carbone et de 5,4 à $8,0 \% 0$ $(\mathrm{Me}=6,0 \%$; IIQ $=[5,7 ; 7,0])$ pour l'azote $(n=7)$. Ces valeurs sont en accord avec la fréquentation d'un environnement terrestre tempéré à végétation de type $\mathrm{C}_{3}$ (Bocherens, 1997). Bien qu'aucune différence statistique significative entre les espèces n'ait pu être mise en évidence $(p=0,057$ et $p=0,133$, pour le carbone et l'azote respectivement), potentiellement en raison du manque de puissance des tests due aux faibles effectifs, on note toutefois que les bovins ont des valeurs de carbone plus faibles et d'azote plus élevées que les autres espèces (tableau 1, fig. 2). S'il est difficile de proposer des interprétations avec si peu de données, on peut cependant suggérer que les bovins se soient nourris ou aient été affouragés par des végétaux pouvant provenir de milieux plus fermés (avec valeurs de $\delta^{13} \mathrm{C}$ plus basses; Van der Merwe et Medina, 1991; Rodière et al., 1996) et de zones où les conditions édaphiques et/ou le type de végétation diffèrent de celles fréquentées par les ovicaprins et les porcins (e.g. Drucker et al., 2003 et 2011). L'observation de différences isotopiques entre les bovins et ovicaprins dans cette région n'est pas singulière. L'étude de plusieurs sites de l'âge du Bronze dans la région de Clermont-Ferrand suggère des différences alimentaires entre ces deux espèces, pouvant peut-être résulter de transhumances bovines et/ou de stabulations hivernales ovicaprines (Le Bras-Goude et al., 2011).

Chez les sujets humains les valeurs isotopiques s'étendent de $-20,9$ à $-18,9 \%$ o $(\mathrm{Me}=-20,2 \%$; IIQ $=[-20,4 ;-19,9])$ et de 8,6 à 13,0\%o $(\mathrm{Me}=10,3 \%$; IIQ $=[9,8 ; 10,8])$ pour le carbone et l'azote respectivement $(\mathrm{n}=58)$. La variation isotopique enregistrée au sein de la population humaine est particulièrement importante en ce qui concerne l'azote $\left(\Delta \delta^{15} \mathrm{~N}=4,4 \%\right.$, contre $2,6 \%$ o au sein de la faune) et suggère une différenciation intrapopulationnelle dans la consommation des protéines, avec un accès plus ou moins important aux ressources animales selon les individus. La comparaison entre les données isotopiques de la faune et du groupe humain $\left(\Delta \delta^{13} \mathrm{C}_{\text {humain-faune }}=1,3 \% ; \Delta \delta^{15} \mathrm{~N}_{\text {humain-faune }}=4,1 \%\right.$ ) souligne l'importance des protéines animales (viande, produits secondaires, voire poisson) dans l'alimentation générale de cette population (fig. 2).

Lorsque le modèle théorique des relations trophiques est appliqué (cf. § Méthodologie), nous observons : 
1) qu'une majorité des individus auraient consommé une très grande quantité de viande d'herbivores issue des ressources locales, 2) que certains auraient pu consommer d'autres ressources protéiniques enrichies en azote-15 et 3) que deux sujets (sép. 30 et 67), présentant les valeurs les plus basses de $\delta^{15} \mathrm{~N}$, se distinguent du reste du groupe et pour lesquels une consommation moins importantes en protéines animales (au profit des ressources végétales) pourrait être proposée (fig. 3). Une consommation de $100 \%$ de protéines animales terrestres n'étant pas envisageable, il semblerait que d'autres ressources enrichies en azote-15, c'est-à-dire de niveau trophique plus élevé, soient consommées par les individus présentant les valeurs de $\delta^{15} \mathrm{~N}$ les plus hautes. Parmi ces ressources, on peut citer les poissons d'eau douce, voire des espèces anadromes. La figure 3 montre un exemple de distribution des valeurs de $\delta^{13} \mathrm{C}$ et $\delta^{15} \mathrm{~N}$ archéologiques disponibles dans la littérature pour le brochet et le saumon et les valeurs attendues pour des consommateurs exclusifs de ces ressources. Cette approche, aussi empirique soit-elle, nous permet de proposer l'hypothèse que des poissons, tels que le brochet, le saumon, la lamproie ou la truite (actuellement présents dans le réseau aquatique auvergnat; Brugel et al., non publié), ont potentiellement pu être consommés en quantité non négligeable par certains sujets de Pontcharaud. L'étape suivante de notre analyse est donc de tenter de repérer des " patrons alimentaires » au sein de cette population et de voir si ces derniers sont en relation avec les critères anthropologiques et archéologiques retenus dans cette étude.

\section{RELATION ENTRE LES PRATIQUES ALIMENTAIRES ET LES PARAMETRES BIOLOGIQUES ET ARCHÉOLOGIQUES}

T es informations sur l'âge au décès des adultes et des _ujets immatures, le sexe et la stature des adultes figurent dans le tableau 1. Les individus pour lesquels le collagène est bien préservé et les valeurs isotopiques interprétables se répartissent en un groupe adulte comprenant seize femmes, quinze hommes et six sujets de sexe indéterminé et en un groupe d'individus immatures de différentes classes d'âge comprenant sept enfants en bas âge (0-4 ans), dix enfants (5-14 ans) et quatre adolescents (15-19 ans). Lorsque l'âge a pu être déterminé sur les sujets adultes, ces derniers ont été répartis dans

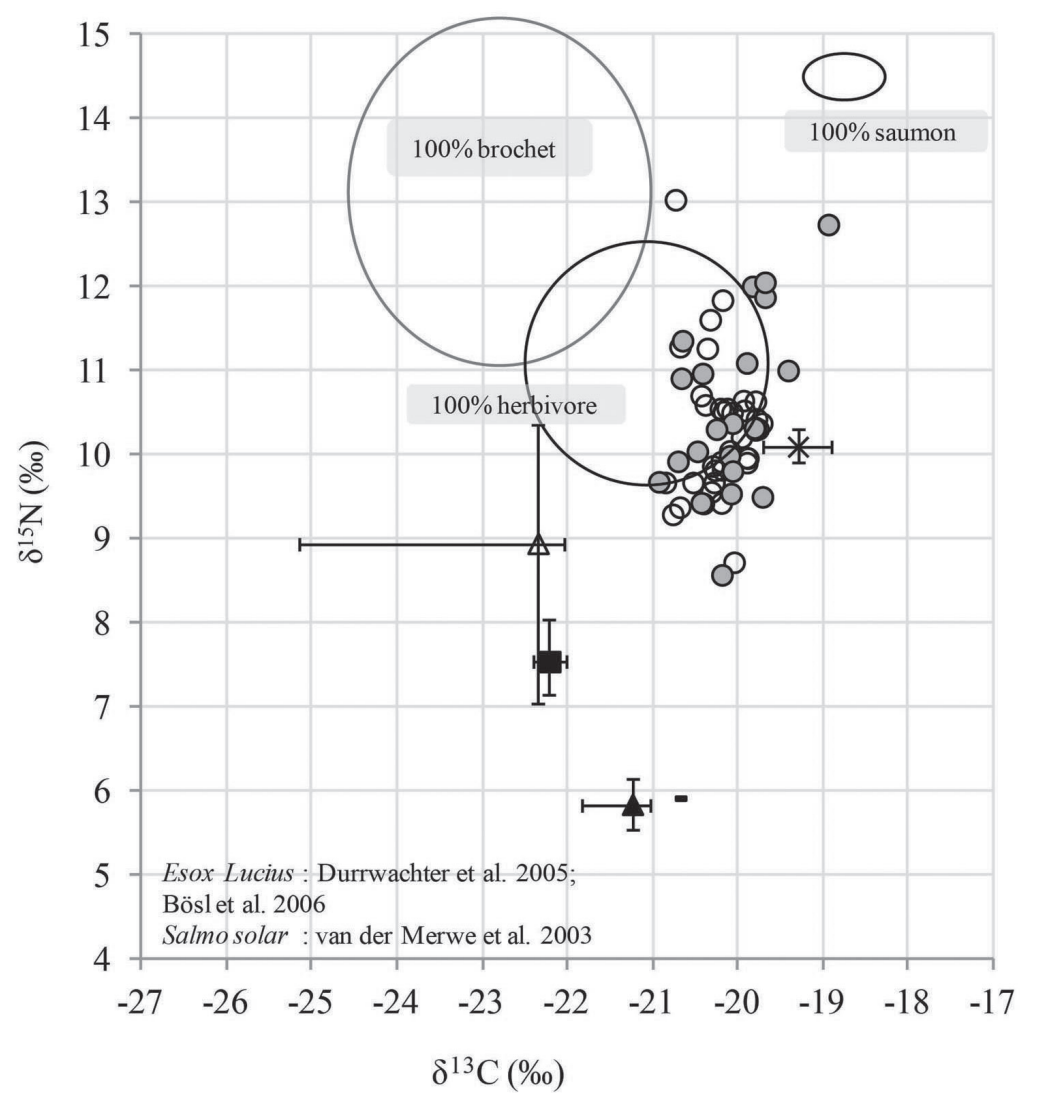

O Humain adulte $(\mathrm{n}=37)$

OHumain immature $(n=21)$

- Suiné ( $\mathrm{n}=1)$

Bovin $(n=2)$

Médiane et percentiles 2,5 et 97,5

Fig. 3 - Distribution des valeurs isotopiques $\left(\delta^{13} \mathrm{C}, \delta^{15} \mathrm{~N}\right)$ des sujets humains de Pontcharaud 2 avec les médianes et les percentiles $(2,5$ et 97,5) de la faune du site et de l'ichtyofaune choisies pour la comparaison. Représentation graphique de la relation théorique proieprédateur.

Fig. 3 - Human $\delta 13 C$ and $\delta 15 N$ values from Pontcharaud 2, faunal isotopic data (median and percentiles: 2.5 and 97.5) from Pontcharaud and from published fish values chosen for comparison. Prey-predator theoretical relationship. 
trois groupes : les adultes jeunes (20-39 ans; $\mathrm{n}=15)$, les adultes matures (40-59 ans; $\mathrm{n}=12)$ et les adultes âgés $(>60$ ans $; n=4)$. La stature nous a également paru être un critère biologique intéressant à prendre en compte pour notre étude. En effet, les modalités de croissance et la stature d'un individu adulte sont conditionnées par plusieurs facteurs, parmi lesquels l'alimentation peut avoir un rôle important (Mafart, 1989; Polet et Orban, 2001); c'est d'ailleurs pour ces raisons que la relation entre le régime alimentaire et la stature est un sujet primordial aujourd'hui dans les études de veille sanitaire et épidémiologiques (Susanne, 1993; Ulijasnek, 2003). Par exemple, une étude menée sur des populations actuelles des îles Cook a montré qu'il existe un accroissement de la taille au fil des générations s'effectuant parallèlement à une évolution des modes de subsistance et plus particulièrement marquée par une augmentation des quantités de viande consommées (Ulijasnek, 2003). Le lien entre une consommation de ressources carnées et une stature élevée a également été mis en évidence dans plusieurs populations, comme chez certains chasseurs-cueilleurs de Patagonie (Soto-Heim, 2001). Un tel lien entre la stature et les valeurs de $\delta^{15} \mathrm{~N}$ a par ailleurs aussi été mise en évidence dans une série française de la fin du Moyen Âge (Herrscher et al., 2001). Á Pontcharaud 2, nous avons choisi une comparaison directe entre la stature estimée (en $\mathrm{cm}$ ) et les données isotopiques par le biais d'analyses de corrélation, en prenant soin de séparer les sujets féminins et masculins.

La comparaison des valeurs isotopiques entre les sujets féminins et masculins ne montre pas de différence significative $(p=0,535$ et $p=0,899$, pour le carbone et l'azote respectivement). De même, ces valeurs ne sont pas corrélées avec la stature $(r h o=0,146$ et $r h o=$ 0,138 , pour le carbone et l'azote respectivement). En revanche, l'âge au décès semble être un paramètre biologique influençant la distribution des valeurs isotopiques ( $p<0,001$ et $p=0,009$, respectivement pour le carbone et l'azote; tableau 2). En effet, les comparaisons par classe d'âge 2 à 2 montrent que le groupe des immatures âgés de

\begin{tabular}{|c|c|c|c|c|c|c|c|c|c|c|c|}
\hline \multirow{2}{*}{ Classe d'âge } & \multirow{2}{*}{$\mathbf{N}$} & \multicolumn{5}{|c|}{$\delta^{13} \mathrm{C}(\% 0)$} & \multicolumn{5}{|c|}{$\delta^{15} \mathrm{~N}(\% 0)$} \\
\hline & & Min & Max & Médiane & IIQ & p* & Min & Max & Médiane & IIQ & p* \\
\hline Sexe & & & & & & 0,535 & & & & & 0,899 \\
\hline Homme & 15 & $-20,9$ & $-19,7$ & $-20,1$ & {$[-20,5 ;-19,8]$} & & 9,3 & 11,3 & 10,3 & {$[9,7 ; 10,5]$} & \\
\hline Femme & 16 & $-20,7$ & $-19,8$ & $-20,2$ & {$[-20,4 ;-20,0]$} & & 8,7 & 13,0 & 9,9 & {$[9,7 ; 10,6]$} & \\
\hline Classe d'âge & & & & & & & & & & & \\
\hline En 2 classes & & & & & & 0,465 & & & & & 0,405 \\
\hline Immatures & 21 & $-20,9$ & $-18,9$ & $-20,1$ & {$[-20,4 ;-19,8]$} & & 8,6 & 12,7 & 10,3 & {$[9,8 ; 11,1]$} & \\
\hline Adultes & 37 & $-20,9$ & $-19,7$ & $-20,2$ & {$[-20,4 ;-19,9]$} & & 8,7 & 13,0 & 10,3 & {$[9,8 ; 10,6]$} & \\
\hline En 6 classes & & & & & & $<0,001$ & & & & & 0,008 \\
\hline Bébé & 7 & $-19,9$ & $-18,9$ & $-19,7$ & {$[-19,8 ;-19,4]$} & & 10,3 & 12,7 & 11,9 & {$[11,0 ; 12,1]$} & \\
\hline Enfant & 10 & $-20,9$ & $-19,7$ & $-20,5$ & {$[-20,7 ;-20,2]$} & & 8,6 & 11,4 & 10,0 & {$[9,5 ; 10,9]$} & \\
\hline Adolescent & 4 & $-20,3$ & $-20,1$ & $-20,1$ & - & & 9,5 & 10,3 & 9,9 & - & \\
\hline Adulte jeune & 15 & $-20,9$ & $-19,8$ & $-20,2$ & {$[-20,4 ;-19,9]$} & & 8,7 & 10,7 & 9,9 & {$[9,7 ; 10,6]$} & \\
\hline Adulte mature & 12 & $-20,8$ & $-19,8$ & $-20,2$ & {$[-20,4 ;-20,1]$} & & 9,3 & 11,8 & 10,3 & {$[9,7 ; 10,9]$} & \\
\hline Adulte âgé & 4 & $-20,1$ & $-19,8$ & $-19,9$ & - & & 9,9 & 10,6 & 10,2 & - & \\
\hline Architecture** & & & & & & 0,476 & & & & & 0,831 \\
\hline Absence & 49 & $-20,9$ & $-19,7$ & $-20,2$ & {$[-20,4 ;-20,0]$} & & 8,6 & 13,0 & 10,1 & {$[9,7 ; 10,6]$} & \\
\hline Présence & 2 & $-20,4$ & $-20,3$ & - & - & & 9,6 & 11,3 & - & - & \\
\hline Type d'inhumation** & & & & & & $<0,001$ & & & & & 0,006 \\
\hline Simple & 39 & $-20,7$ & $-19,7$ & $-20,2$ & {$[-20,3 ;-19,9]$} & & 8,6 & 13,0 & 10,3 & {$[9,8 ; 10,6]$} & \\
\hline Double & 5 & $-20,7$ & $-19,9$ & $-20,2$ & - & & 8,7 & 11,8 & 10,0 & - & \\
\hline Multiple & 7 & $-20,9$ & $-20,4$ & $-20,7$ & {$[-20,9 ;-20,5]$} & & 9,3 & 9,9 & 9,7 & {$[9,4 ; 9,7]$} & \\
\hline
\end{tabular}

* Test de Kruskal-Wallis : approximation de Monte-Carlo (10000 répétitions) si nombre de groupes $>2$; test exact de Wilcoxon si nombre groupes $=2$

** 0-4 ans exclus

- Médiane et/ou IIQ non présentés pour les groupes d'effectif trop faible

Tabl. 2 - Statistiques descriptives des mesures isotopiques selon différents groupes.

Table 2 - Descriptive statistics of stable isotope values according to different groups. 
0 à 4 ans $(\mathrm{n}=7)$ présente des valeurs de $\delta^{13} \mathrm{C}$ et $\delta^{15} \mathrm{~N}$ significativement différentes $(\mathrm{p}<0,05)$ des autres groupes d'immatures et d'adultes, à l'exception des adultes âgés (tableau 3).

Compte tenu de l'âge de ces très jeunes enfants, ces résultats sont probablement dus à l'enregistrement d'un signal lacté (non-sevré, en cours de sevrage). En effet, lors de l'allaitement maternel, l'enfant consomme exclusivement les protéines de sa propre espèce, ce qui lui confère un niveau trophique supérieur et par conséquent un enrichissement en ${ }^{15} \mathrm{~N}$ de ses tissus synthétisés durant cette période. Depuis les travaux de Fogel et al. (1989), de nombreuses études se sont intéressées à ces périodes alimentaires particulières que sont l'allaitement et le sevrage, autant chez l'homme que chez l'animal (Balasse et al., 1997; Katzenberg 1993; Herrscher, 2013). Ces travaux, mais en particulier ceux de Balasse (Balasse et al., 2000; Balasse et Tresset, 2002) et de Herrscher (2003, 2011 et 2013), abordent toutefois l'étude des jeunes individus via une méthodologie spécifique et adaptée (multiéchantillonnage) que nous n'avons pas encore mise en application sur la série de Pontcharaud 2. Par conséquent, pour répondre aux objectifs initialement proposés dans cet article, il nous apparaît indispensable d'écarter

\begin{tabular}{|c|c|c|c|}
\hline & & $\delta^{13} \mathrm{C}(\%)$ & $\delta^{15} \mathrm{~N}(\%)$ \\
\hline \multicolumn{4}{|l|}{ Âge en 6 classes } \\
\hline \multirow[t]{5}{*}{ Bas âge } & Enfant & 0,005 & 0,023 \\
\hline & Adolescent & 0,023 & 0,031 \\
\hline & Adulte jeune & 0,005 & 0,003 \\
\hline & Adulte mature & 0,006 & 0,037 \\
\hline & Adulte âgé & 0,156 & 0,073 \\
\hline \multirow[t]{4}{*}{ Enfant } & Adolescent & 0,159 & 0,899 \\
\hline & Adulte jeune & 0,159 & 0,853 \\
\hline & Adulte mature & 0,191 & 0,808 \\
\hline & Adulte âgé & 0,108 & 0,853 \\
\hline \multirow[t]{3}{*}{ Adolescent } & Adulte jeune & 0,736 & 0,853 \\
\hline & Adulte mature & 0,736 & 0,678 \\
\hline & Adulte âgé & 0,396 & 0,678 \\
\hline \multirow[t]{2}{*}{ Adulte jeune } & Adulte mature & 0,736 & 0,808 \\
\hline & Adulte âgé & 0,159 & 0,808 \\
\hline Adulte mature & Adulte âgé & 0,146 & 0,999 \\
\hline \multicolumn{4}{|c|}{ Type d'inhumation* } \\
\hline \multirow[t]{2}{*}{ Simple } & Double & 0,771 & 0,999 \\
\hline & Multiple & $<0,001$ & 0,003 \\
\hline Double & Multiple & 0,015 & 0,224 \\
\hline
\end{tabular}

* 0-4 ans exclus

Tabl. 3 - Comparaisons 2 à $2: p$-values des tests exacts de Wilcoxon corrigées par le FDR.

Table 3-2 $\times 2$ comparisons: Wilcoxon signed-ranktests $p$-values corrected by FDR. ce groupe d'âge pour la suite de l'analyse des données. Ainsi d'autres comparaisons ont été effectuées, à la fois entre les groupes d'âge immatures (enfant/adolescent), entre les groupes d'âge adultes (jeune/mature/âgé) et entre les immatures et les adultes. Aucun des tests réalisés n'a mis en évidence de différence statistiquement significative (au seuil de $5 \%$ ) des valeurs isotopiques en fonction des groupes d'âge (tableau 3).

Par conséquent, les données biologiques individuelles de la série de Pontcharaud 2, considérées dans cette étude, ne permettent pas d'identifier des « groupes » ayant des comportements alimentaires particuliers, selon le sexe et l'âge des individus. Si ces critères biologiques peuvent induire des règles socio-économiques particulières, en revanche ils ne semblent n'avoir en rien dicté des comportements alimentaires spécifiques ou tout au moins des différences perceptibles dans la nature et la quantité des protéines consommées par les sujets de Pontcharaud 2.

D'un point de vue archéologique, et en particulier des pratiques funéraires, plusieurs critères ont retenus notre attention : l'identification d'une architecture pérenne ou non pour la tombe et le nombre d'individus par tombe (individuelle ou multiple). De la même manière que pour

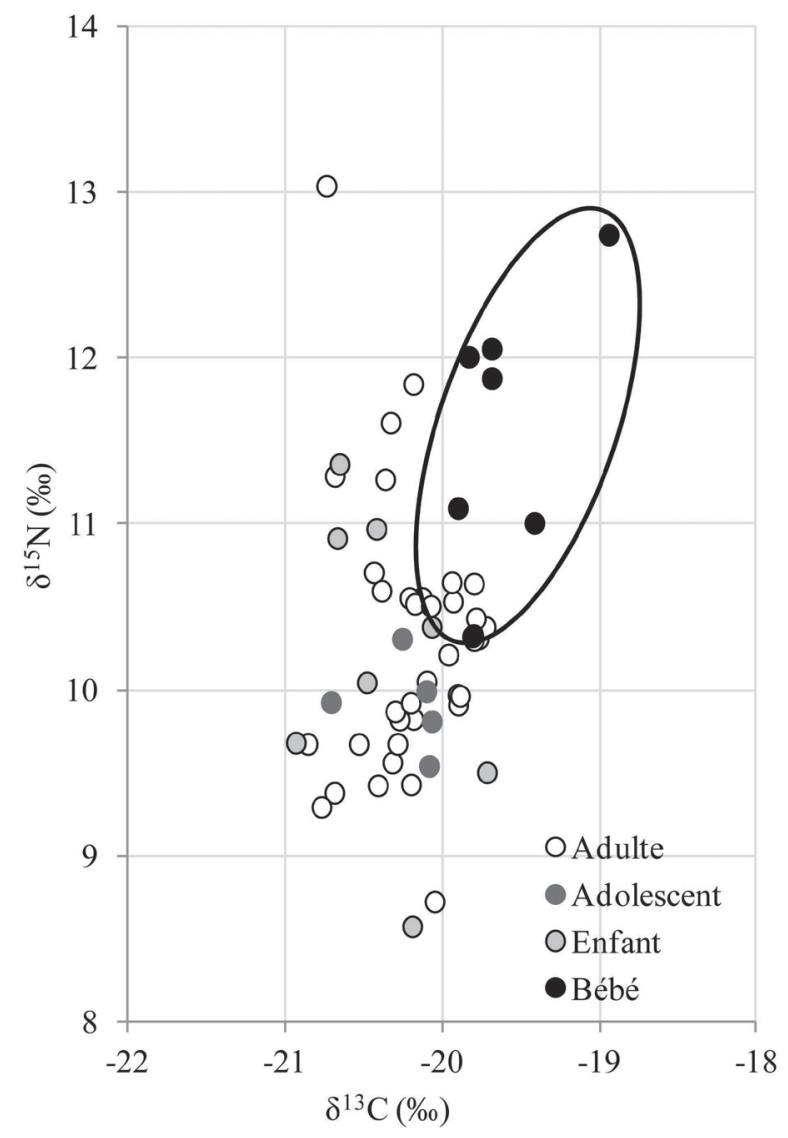

Fig. 4 - Distribution des valeurs isotopiques $(\delta 13 \mathrm{C}, \delta 15 \mathrm{~N}) \mathrm{des}$ sujets humains de Pontcharaud 2 en fonction des groupes d'âge. Le groupe des « bas âges » (0-4 ans) est cerclé.

Fig. 4 - Human stable isotope values according to age groups. "Infant" group (0-4 years old) is circled. 
les données biologiques, ces critères archéologiques ont été croisés avec les valeurs isotopiques (tableau 2). Aucune différence significative des données isotopiques n'a pu être mise en évidence selon le critère architecture pérenne/absence d'architecture $(p=0,476$ et $p=0,831$ pour le carbone et l'azote respectivement). Le dispositif architectural, tout comme le sexe des individus, ne sont donc pas des critères qui, dans cette étude, permettent de distinguer des sujets ayant eu une alimentation différente. En revanche, il existe une différence très significative des valeurs de $\delta^{13} \mathrm{C}$ et $\delta^{15} \mathrm{~N}$ entre les sujets mis au jour dans l'inhumation multiple de sept individus et ceux découverts dans les tombes individuelles (respectivement $\mathrm{p}<0,001$ et $\mathrm{p}=0,003$ ). Parallèlement à ce résultat, aucune différence significative des valeurs de $\delta^{13} \mathrm{C}$ et de $\delta^{15} \mathrm{~N}$ n'est observée entre les sujets des tombes individuelles et ceux des tombes doubles (respectivement $\mathrm{p}=0,771$ et $\mathrm{p}=0,999)$. Ce résultat rejoint les deux présentés précédemment et met en évidence la particularité isotopique des 7 individus de l'inhumation multiple. Les individus de cette structure possèdent en effet les valeurs de $\delta^{13} \mathrm{C}(\mathrm{Me}=-20,7 \% 0 ;$ IIQ $=[-20,9 ;-20,5])$ les plus

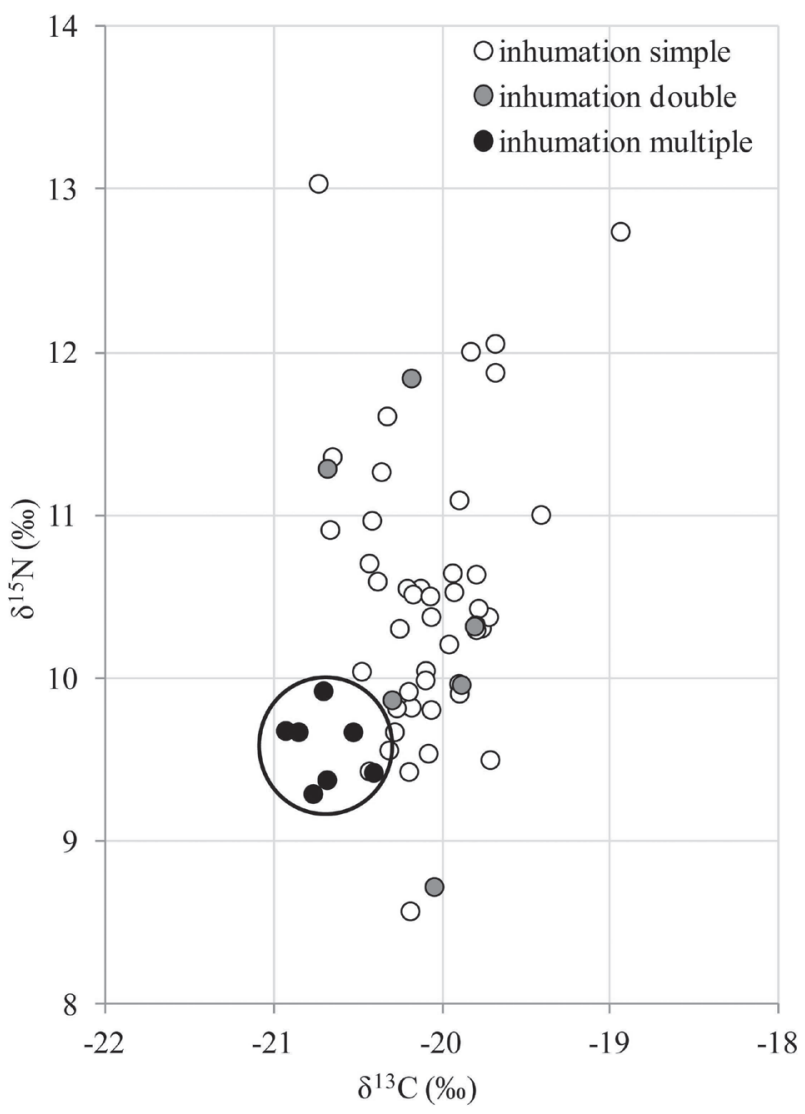

Fig. 5 - Distribution des valeurs isotopiques $\left(\delta^{13} \mathrm{C}, \delta^{15} \mathrm{~N}\right)$ des sujets humains de Pontcharaud 2 en fonction du type d'inhumation (catégorie 0-4 ans exclus). Les individus de la sépulture multiple sont cerclés.

Fig. 5 - Human stable isotope values according to burial type (excl. 0-4 years old). Individuals from the multiple burial are circled. basses du groupe humain et des valeurs de $\delta^{15} \mathrm{~N}$ particulièrement basses également $(\mathrm{Me}=9,7 \%$; IIQ $=[9,4$; 9,7]; fig. 5). Il semblerait donc qu'ils aient, soit consommés moins de protéines animales que les individus inhumés des autres tombes, soit consommés des ressources d'un autre environnement, isotopiquement distinct. Ce dépôt multiple primaire comporte sept sujets. Le statut de ces défunts, comparativement aux autres individus de l'ensemble funéraire, est difficile à définir et ce type d'inhumation a fait l'objet de plusieurs commentaires. Pour Chambon et Leclerc (2007), la position et l'orientation des corps traduiraient une mise en scène à l'intérieur de la fosse distinguant clairement cette inhumation et leurs défunts des autres tombes du site : " une tombe exceptionnelle pour un événement exceptionnel ». Boulestin (2008) note une asymétrie entre les six sujets sur le ventre et le septième déposé sur le côté droit et sur lequel ont été découvertes des dalles de calcaire. Toutefois, pour lui, l'asymétrie n'est pas très marquée et il rejoint la suggestion d'A. Testart : "l'asymétrie se situe plutôt entre le dépôt multiple et la sépulture individuelle 47 qui se situe à proximité » (Testart, 2004) qui corrobore le caractère original de cette sépulture et des sujets qui y sont inhumés. Considérant la particularité de cette structure, la question se pose alors sur le statut et l'origine de ces sujets. Il s'agit en effet d'un regroupement de cinq adultes, uniquement masculins, jeunes ou matures, et deux immatures dont l'âge (7,5-12,5 ans et 12-15 ans) pourrait laisser penser qu'ils ne soient plus socialement considérés comme des enfants. Aucune trace osseuse ne permet d'aller plus loin dans l'interprétation des données, si ce n'est la présence d'une armature de silex dans le rachis de l'un des sujets (Loison, 1998) qui témoignerait d'au moins une mort violente volontaire (Chambon et Leclerc, 2007; Boulestin, 2008).

\section{ESSAI DE COMPARAISON RÉGIONALE}

$\mathrm{P}$ lusieurs sites du Néolithique moyen, contemporains de Pontcharaud 2, ont précédemment fait l'objet d'analyses isotopiques dans une perspective paléoalimentaire. Ces derniers sont situés dans la région languedocienne et garonnaise. Il s'agit des sites du Crès, Béziers, Hérault (Le Bras-Goude et al., 2009; Loison et al., 2004) des Plots, Berriac, Aude, de VilleneuveTolosane - Cugnaux, Toulouse, Haute-Garonne (Gandelin et Vaquer, 2008) et Narbons, Montesquieu-de-Lauragais, Haute-Garonne (Tchérémissinoff, 2003 ; Tcherémissinoff et al., 2005 ; fig. 1 ; tableau 4). Ces deux « régions » languedocienne et garonnaise correspondent à des aires géographiques bien délimitées les unes des autres, séparées d'un côté et de l'autre par le seuil de Naurouze. Une partie des sites (Languedoc) se trouve dans la plaine de l'Aude et proche de la Méditerranée, l'autre partie (Garonne) est située en Haute-Garonne, dans un relief collinéen au climat tempéré sous influence océanique (actuellement). Localisé au sud de Toulouse, le site de 
Villeneuve-Tolosane - Cugnaux présente plusieurs centaines de structures du Néolithique moyen, quatre grands systèmes d'enceintes et une vingtaine de structures ayant livré des restes humains (Brossier et Marlière, 2000; Gandelin, 2011) dont quatre sont contemporaines de Pontcharaud 2. Le site de Narbons se situe au sud-est de Montesquieu-de-Lauragais. L'opération archéologique a mis au jour six fosses profondes, peut-être des puits, attribuées au Néolithique moyen dont quatre contenaient des vestiges humains (Tchérémissinoff et al., 2005). Les datations radiométriques placent chronologiquement deux structures dans la deuxième moitié du $\mathrm{V}^{\mathrm{e}}$ millénaire. Sur ces sites garonnais, dix-sept sujets humains et vingt et un restes de faune ont pu être analysés, témoignant d'une alimentation mixte (végétale et animale) mais avec une part importante de protéines animales, voire de poisson pour certains individus (Herrscher et Le Bras-Goude, 2008). En Languedoc, le site des Plots situé à Berriac comporte 104 structures en creux dont quarante-six ont livré du matériel de type Chasséen ancien garonnais qui permet de les attribuer à la seconde moitié du $\mathrm{V}^{\mathrm{e}}$ millénaire. Six d'entre elles ont reçu des inhumations (Duday et Vaquer 2003). Le site du Crès, au nord de Béziers, est composé de 189 structures en fosse, domestiques et funéraires. Les datations radiométriques et par le mobilier céramique indiquent une occupation comprise entre 4350-4000 BC (Loison et al., 2004; Loison et Schmitt, 2009). Les analyses isotopiques ont permis de mettre en évidence une consommation de protéines majoritairement tournée vers les ressources agricoles comparativement aux ressources pastorales et de souligner l'absence des ressources marines dans l'alimentation malgré la proximité de la mer (Le Bras-Goude et al., 2009).

L'étude comparative déjà réalisée entre ces deux zones géographiques susmentionnées avait mis en évidence des différences à la fois environnementales et économiques dans les modes de subsistance des groupes humains de chaque région, avec une consommation alimentaire plus tournée vers la viande et les produits secondaires chez les individus issus des sites de Garonne et plus tournée vers des ressources mixtes, incluant une forte proportion de céréales, pour ceux issus du Languedoc (Herrscher et Le Bras-Goude, 2008 et 2010). Toutefois, dans la comparaison inter-régionale réalisée ici, seules les inhumations strictement attribuées au même intervalle chronologique que celui de Pontcharaud 2, par le radiocarbone (fig. 6) et/ou par le mobilier, ont été prises en compte, expliquant que le corpus de comparaison repose sur vingtcinq individus adultes et immatures supérieurs à 4 ans (tableau 4). Les sujets inhumés sans matériel strictement attribuable à la deuxième moitié du $\mathrm{V}^{\mathrm{e}}$ millénaire et non datés ont été exclus. Par ailleurs, ce travail intègre bien entendu le fait que les sujets étudiés proviennent d'unités funéraires (sélection d'individus) et qu'ils ne sont pas forcément représentatifs de l'ensemble de la population vivante. Ce biais, inhérent à tous les travaux archéoanthropologiques, nous invite à la plus grande prudence concernant les résultats et les hypothèses formulées.

La figure 7 présente l'ensemble des données isotopiques de la faune et des humains inclus dans cette comparaison (tous les sujets de moins de 4 ans ont été exclus); le tableau 4 récapitule les données générales de chacun des sites et le nombre d'échantillons humains et animaux pris en compte. La position trophique des groupes humains par rapport à la faune, propre à chacun des sites (herbivores domestiques et porcins : $\Delta \delta^{15} \mathrm{~N}$, est différente selon les régions). Le groupe humain de Pontcharaud 2 se distingue statistiquement des autres régions considérées (tableau 5, fig. 7), avec une consommation de protéines animales plus importante et/ou une consommation importante de protéines riches en ${ }^{15} \mathrm{~N}$ relativement aux sujets des autres régions (e. g. poissons, animaux non-sevrés). La position plus élevée de Pontcharaud 2 dans la chaîne trophique par rapport à la Garonne pourrait s'expliquer 1) par la présence un peu plus importante de ressources aquatiques dans l'alimentation et/ou 2) par la consommation de jeunes animaux non sevrés. L'altitude (quoique modérée), le relief plus vallonné du Massif central, un climat plus contrasté/continental, comparativement au Languedoc et à la Garonne, pourraient-ils conduire le groupe humain de Pontcharaud 2 à exploiter toutes les différentes zones écologiques proches, qui lui permettraient d'optimiser l'acquisition des ressources alimentaires, incluant le réseau fluviatile à proximité du site et en altitude? Par ailleurs, les jeunes animaux non sevrés sont des ressources alimentaires qui ont également des valeurs de $\delta^{15} \mathrm{~N}$ élevées en raison de leur position plus haute dans la chaîne alimentaire (Schurr, 1998). Leur

\begin{tabular}{|l|c|c|c|c|c|l|}
\hline Site & Région & Type de site & Altitude & Humain & Animal & Références \\
\hline Le Crès & Languedoc & Domestique & $20 \mathrm{~m}$ & 18 & 7 & Loison et al., 2004; Loison et Schmitt, 2009 \\
\hline Les Plots & Languedoc & Domestique & $101 \mathrm{~m}$ & 1 & 4 & Duday et Vaquer, 2003 \\
\hline $\begin{array}{l}\text { Villeneuve-Tolosane } \\
\text { - Cugnaux }\end{array}$ & Garonne & Domestique & $162 \mathrm{~m}$ & 4 & 14 & Gandelin et Vaquer, 2008 \\
\hline Narbons & Garonne & Funéraire & $206 \mathrm{~m}$ & 2 & 1 & Tchérémissinoff et al., 2005 \\
\hline Pontcharaud 2 & Auvergne & Funéraire & $335 \mathrm{~m}$ & 51 & 7 & Loison, 1998; cette étude \\
\hline
\end{tabular}

Tabl. 4 - Synthèse générale des données archéologiques et géographiques des échantillons retenus pour la comparaison interrégionale. Table 4 - Synthetic archaeological and geographical data, and the number of human and faunal analyses from sites used for the regional comparison study. 


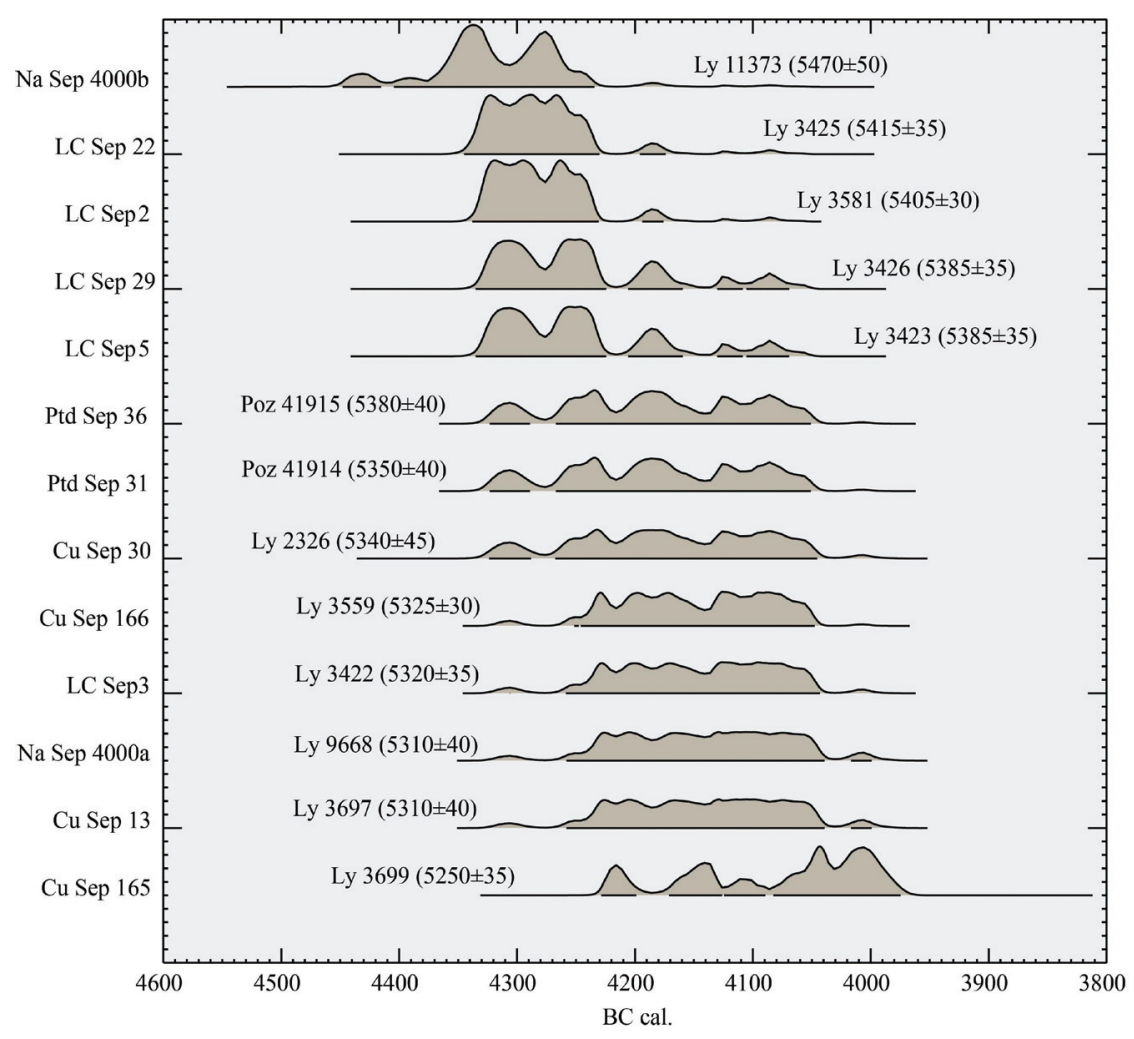

Fig. 6 - Datations radiocarbones (logiciel Calib Rev 6.1.0; Stuiver et Reimer, 1993) de sépultures de la Garonne (Cu et Na : Gandelin et Vaquer, 2008; Tchérémissinoff et al., 2005) et du Languedoc (LC : Loison et Schmitt, 2009) prises en compte dans cette étude.

Fig. 6 - Radiocarbon dates (Calib Rev 6.1.0 software; Stuiver \& Reimer, 1993) from Garonne and Languedoc burials (Cu and Na: Gandelin \& Vaquer, 2008; Tchérémissinoff \& al., 2005; LC: Loison \& Schmitt, 2009) used in the comparison study.

consommation peut donc entraîner une augmentation des valeurs de $\delta^{15} \mathrm{~N}$ dans les tissus des consommateurs, par rapport à ceux qui consomment de la viande d'animaux matures. L'absence d'informations archéozoologiques, sur la faune terrestre et sur l'ichtyofaune (non présente ou non recherchée au moment des fouilles du site), ne permet pas d'aller plus loin dans ces hypothèses.

Les sites du Languedoc diffèrent de manière significative ( $\mathrm{p}<0,05$; tableau 5 ) de ceux des autres zones géographiques : consommation moindre de protéines animales et acquisition des ressources dans un espace plus ouvert/ défriché et, d'autant plus, si l'on écarte le sujet particulier (fléché sur la figure 8). On remarque également que la variabilité isotopique intra-groupe (notamment de l'azote, $\Delta \delta^{15} \mathrm{~N}$ ) est plus importante au sein des sujets de Pontcharaud $(4,3 \%$, et $3,0 \%$ si on exclut l'individu particulièrement haut en ${ }^{15} \mathrm{~N}$ ) que pour ceux de la Garonne et du Languedoc, indiquant que les groupes ne sont pas homogènes dans leurs choix alimentaires (surtout à Pontcharaud 2). Dans chacune de ces régions, les groupes humains ont

\begin{tabular}{|l|c|c|c|c|c|c|c|c|c|c|c|}
\hline Région & & \multicolumn{9}{|c|}{$\delta^{13} \mathrm{C}(\%)$} & \multicolumn{5}{c|}{$\delta^{15} \mathrm{~N}(\%)$} \\
\hline & $\mathrm{N}$ & Min. & Max. & Moy. (SD) & Médiane [IIQ] & $\mathrm{p}^{*}$ & Min. & Max. & Moy. (SD) & Médiane [IIQ] & $\mathrm{p}^{*}$ \\
\hline Auvergne & 51 & $-20,9$ & $-19,7$ & $-20,2(0,3)$ & $\begin{array}{c}-20,2 \\
{[-20,4 ;-20,0]}\end{array}$ & $<0,001$ & 8,6 & 13,0 & $10,2(0,8)$ & $\begin{array}{c}10,1 \\
{[9,7 ; 10,6]}\end{array}$ & $<0,001$ \\
\hline Garonne & 6 & $-20,9$ & $-19,9$ & - & $\begin{array}{c}-20,8 \\
{[-]}\end{array}$ & & 8,4 & 10,4 & - & 9,3 \\
{$[-]$} & $\begin{array}{c}8,1 \\
{[7,7 ; 8,5]}\end{array}$ \\
\hline Languedoc & 19 & $-20,6$ & $-18,6$ & $-19,4(0,6)$ & $\begin{array}{c}-19,3 \\
{[-19,8 ;-19,0]}\end{array}$ & & 6,8 & 11,1 & $8,2(0,9)$ & \\
\hline
\end{tabular}

* Test de Kruskal-Wallis, approximation de Monte-Carlo (10000 répétitions)

Tests exacts de Wilcoxon pour comparaisons 2 à 2 ( $p$-values corrigées par la méthode du FDR) :

$-\delta^{13} \mathrm{C}:$ Auvergne / Garonne $: \mathrm{p}=0,055$; Auvergne / Languedoc $: \mathrm{p}<0,001 ;$ Garonne / Languedoc $: \mathrm{p}<0,001$

$-\delta^{15} \mathrm{~N}$ : Auvergne / Garonne $: \mathrm{p}=0,030 ;$ Auvergne / Languedoc $: \mathrm{p}<0,001 ;$ Garonne / Languedoc $: \mathrm{p}=0,004$

Tabl. 5 - Statistiques descriptives des mesures isotopiques pour les sujets considérés dans l'analyse comparative régionale.

Table 5 - Descriptive statistics of stable isotope values for individuals included in the regional comparative analysis. 
la possibilité d'acquérir leurs ressources alimentaires par l'élevage, la chasse, la culture des céréales et la pêche en eau douce (et en mer pour le Languedoc). Le choix dans l'acquisition de ces ressources est variable d'une zone géographique à l'autre (fig. 7), et peut impliquer des paramètres comme l'environnement local, l'altitude, ou bien des paramètres culturels difficiles à définir (par exemple un recrutement spécifique au sein de la nécropole). Certes le site de Pontcharaud 2 n'est pas un site d'altitude ou de montagne comme on peut le définir habituellement, mais comme le souligne de Garine (2007), même les accidents de terrain de faibles altitudes peuvent être considérés comme des «montagnes » par rapports aux plaines environnantes selon les conceptions culturelles. Par ailleurs, la chaîne des Puys offre, à moins de $10 \mathrm{~km}$ à vol d'oiseau du site, des terrains de plus de 1000 m d'altitude, probablement exploités au Néolithique. Des études diachroniques et actuelles ont mis en évidence des

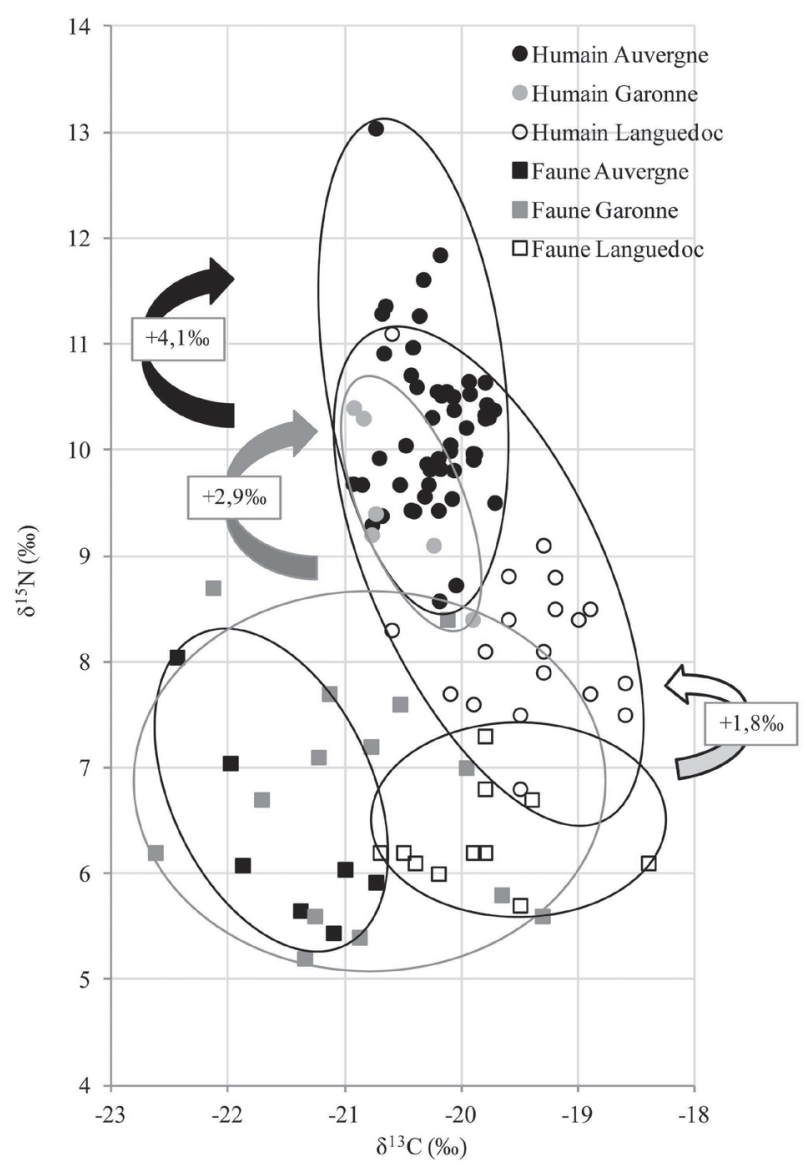

Fig. 7 - Distribution des valeurs isotopiques $\left(\delta^{13} \mathrm{C}, \delta^{15} \mathrm{~N}\right)$ des sujets humains et de la faune des 3 régions considérées : Languedoc, Garonne et Auvergne (catégorie 0-4 ans exclus pour tous les sites). Les flèches informent sur le niveau trophique des groupes humains par rapport à la faune de chaque région ( $\Delta \delta 15$ Nhumain-faune).

Fig. 7 - Distribution of human and animal stable isotope values from the 3 regions studied: Languedoc, Garonne and Auvergne (excl. 0-4 yrolds). Arrows indicate the trophic position of humans compared to animals for each region ( $\triangle \delta 15$ Nhuman-fauna). particularismes alimentaires liés aux milieux d'altitude et ont notamment montré la dominance des activités pastorales pour la production de lait et de fromage surtout, mais également de viande (potentiellement fumée pour le transport), aliments riches en protéines (Garine, 2007). De même, des travaux menés sur une population archéologique médiévale dans les Alpes françaises étayent également l'hypothèse proposée pour Pontcharaud 2, à savoir une consommation plus importante de protéines animales par les groupes d'altitude comparativement à ceux vivant en plaine (Bailly-Maitre et al., 2008; Herrscher et al., 2009). L'ensemble de ces résultats isotopiques montrent que le site de Pontcharaud 2 se distingue à la fois par son environnement local et les ressources disponibles et par les besoins énergétiques nécessaires à la population pour s'adapter à son environnement (e. g. climat, morphologie du territoire).

Une « constante » semble également se dessiner de cette comparaison géographique avec 1) la présence d'individus ayant des valeurs de $\delta^{15} \mathrm{~N}$ élevées et de $\delta^{13} \mathrm{C}$ moyennes ou plus basses que le reste du groupe ou à l'inverse des sujets ayant des valeurs de $\delta^{15} \mathrm{~N}$ relativement basses et 2) des individus de sexe féminin délimitant la variabilité intra-groupe (fig. 8). En Languedoc, ces sujets proviennent du site du Crès, en particulier la femme de la sépulture $10 \mathrm{~b}$ dont l'alimentation est nettement différente et plus riche en protéines animales que celle des autres individus. En Garonne, c'est le sujet féminin de la sépulture 13 de Cugnaux, âgé, atteint de lésions osseuses particulières (tuberculose?) et dont le matériel funéraire est similaire à celui des tombes catalanes (Gandelin et Vaquer, 2008) qui a retenu notre attention, avec une valeur de $\delta^{15} \mathrm{~N}$ relativement basse par rapport aux autres individus (consommation moindre de protéines animales) mais dans la variabilité isotopique mise en évidence en Languedoc au Crès (Herrscher et Le Bras-Goude, 2010). Sur le site de Pontcharaud 2, le sujet féminin de la sépulture 62 présente la valeur la plus haute de $\delta^{15} \mathrm{~N}$ et une valeur de $\delta^{13} \mathrm{C}$ relativement basse alors que les sujets féminin et immature des sépultures 30 et 67 respectivement présentent les valeurs isotopiques en azote les plus basses. Ces sujets ont des valeurs isotopiques similaires à l'individu 13 de Cugnaux et qui sont dans la variabilité des valeurs enregistrées au Crès. Deux hypothèses pourraient expliquer ces observations : soit une diversité alimentaire plus importante chez les individus féminins que masculins en raison de critères socio-économiques, soit une mobilité (géographique) plus importante des sujets féminins qui pourraient par conséquent être plus exposés à des ressources alimentaires isotopiquement plus variées. Plus récemment, la question de la mobilité des sujets du Néolithique moyen a été abordée par d'autres outils (analyses isotopiques du strontium sur l'émail dentaire et l'os; étude préliminaire menées sur Cugnaux, Narbons, Le Crès et Les Plots). Les premiers résultats seraient en faveur d'une mobilité de certains individus au sein des groupes (au cours de leur vie), mais pas nécessairement liée au sexe du sujet (Goude et al., accepté). Toutefois, l'augmentation du nombre de sujets à analyser 


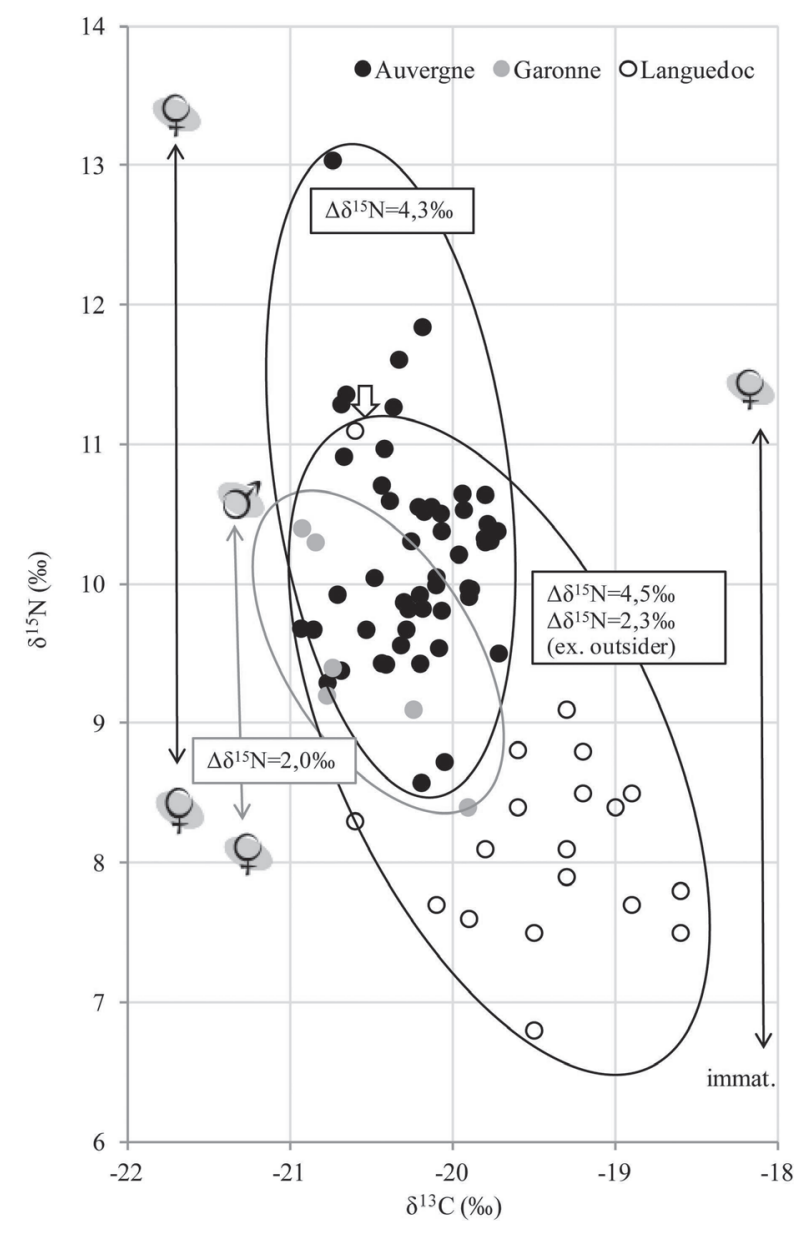

Fig. 8 - Distribution des valeurs isotopiques $\left(\delta^{13} \mathrm{C}, \delta^{15} \mathrm{~N}\right)$ des sujets humains de chaque région et variabilité isotopique de l'azote intra-groupe $\left(\Delta \delta^{15} \mathrm{~N}\right)$.

Fig. 8 - Human stable isotope values from the 3 regions studied and $\delta^{15} \mathrm{~N}$ intra-group variability $\left(\Delta \delta^{15} \mathrm{~N}\right)$.

est nécessaire pour aborder de façon plus pertinente cette question de mobilité des sujets néolithiques.

Cette comparaison interrégionale de 76 sujets de la seconde moitié du $\mathrm{V}^{\mathrm{e}}$ millénaire confirme la présence d'une économie de subsistance régionale avec : 1) des groupes humains plus sédentaires, dont la subsistance est orientée majoritairement vers les produits issus de l'agriculture, fréquentant des environnements de plaine et consommant peu ou pas de ressources aquatiques, et 2) des groupes plus mobiles, avec une subsistance basée majoritairement sur les produits issus de l'élevage, fréquentant des environnements vallonnés continentaux à l'intérieur des terres et pouvant potentiellement consommer plus de ressources aquatiques.

\section{CONCLUSION}

L es recherches isotopiques et anthropologiques réalisées sur cinquante-huit squelettes humains mis au jour dans la nécropole du site de Pontcharaud 2 ont permis d'obtenir de nouvelles informations sur l'ali- mentation de ce groupe humain daté du Néolithique moyen en Auvergne. L'exploitation des ressources vivrières semble être principalement tournée vers les produits carnés (viande, lait, fromage) pour l'ensemble du groupe humain et pourrait être, en partie, dirigée vers les ressources sauvages aquatiques pour certains des sujets, sans qu'il soit possible de définir des groupes sur la base de critères tels que le sexe et l'âge des sujets. En revanche, Pontcharaud 2 est le premier exemple pour lequel une relation a pu être établie entre les pratiques alimentaires et un type de dépôt particulier (que ce soit une sépulture ou non) au sein d'un même site (une analyse inter-site présente des résultats comparables pour le Sud de la France : Le Bras-Goude et al., 2012). En effet, la présence du dépôt multiple, phénomène assez marginal pour la période, et les individus qui y sont inhumés (des hommes adultes, dont un qui porte les traces d'un acte violent à son encontre, et deux enfants), laissent penser qu'il s'agit bien d'un groupe social particulier. L'analyse paléoalimentaire étaye cette hypothèse, témoignant d'une alimentation plus pauvre en produits carnés et/ou d'une origine exogène pour ces sujets. La comparaison interrégionale, menée sur des sujets strictement attribués à la deuxième moitié du Ve millénaire par des datations radiocarbone, conforte l'observation précédemment effectuée (Herrscher et Le BrasGoude, 2010) sur la variété des patrons alimentaires selon les régions et l'impact de l'environnement sur toute la chaine trophique locale. D'autres sites du Néolithique moyen en cours d'étude dans le Languedoc et la vallée du Rhône tel que Le Pirou, Valros ( Loison et al., 2011) devraient permettre d'éclaircir très prochainement les hypothèses proposées ici. Par ailleurs, des études pluridisciplinaires menées sur la série ostéologique de Pontcharaud 2 permettront d'en préciser les modes de vie.

Remerciements : Les auteurs remercient la fondation Nestlé France pour le financement du projet, F. Letterlé (SRA Auvergne) pour l'accès à la collection et l'autorisation d'échantillonnage, ainsi que les reviewers pour leurs corrections et leurs commentaires.

\section{NOTES}

(1) ACR 2004-2007 « Espaces et expressions funéraires au Néolithique moyen dans le monde pyrénéen et ses marges » dir. J. Vaquer.

(2) Projet de la fondation Nestlé France 2009-2011 « Évolution des comportements alimentaires au cours du Néolithique et de l'âge du Bronze dans le Centre et le Sud de la France : apport des marqueurs isotopiques $\left(\delta^{13} \mathrm{C}, \delta^{15} \mathrm{~N}\right) »$, dir. G. Goude et E. Herrscher.

(3) Les standards utilisés par Iso-Analytical sont le foie de bœuf (IA-R042), et d'autres standards internes calibrés par rapport aux standards internationaux IAEA (IA-R045, sulfate d'ammonium; IA-R005 : betterave à sucre; IA-R006 : canne à sucre). 


\section{RÉFÉRENCES BIBLIOGRAPHIQUES}

Ambrose S. H. (1990) - Preparation and Characterisation of Bone and Tooth Collagen for Isotopic Analysis, Journal of Archaeological Science, 17, p. 431-451.

Balasse M., Bocherens H., Tresset A., Mariotti A., Vigne J.-D. (1997) - Émergence de la production laitière au Néolithique? Contribution de l'analyse isotopique d'ossements de bovins archéologiques, Comptes rendus de l'Académie des sciences. Série 2, Sciences de la terre et des planètes, 325, p. 1105-1010.

Balasse M., Tresset A. (2002) - Early Weaning of Neolithic Domestic Cattle (Bercy, France) Revealed by Intra-tooth Variation in Nitrogen Isotope Ratios, Journal of Archaeological Science, 29, 8, p. 853-859.

Balasse M., Tresset A., Bocherens H., Mariotti A., VIGNE J.-D. (2000) - Un abattage «post-lactation » sur des bovins domestiques néolithiques. Étude isotopique des restes osseux du site de Bercy (Paris, France), Ibex Journal of Mountain Ecology - Anthropozoologica, 5, 31, p. 39-48.

Bailly-Maître M., Martin L., Herrscher E., HerVIEU P. (2008) - Se nourrir en haute montagne aux XIII et $\mathrm{XIV}^{\mathrm{e}}$ siècles. Le village de Brandes (Huez, Isère) : apports comparés de l'archéologie, des textes, de l'archéobotanique et de l'anthropologie, Histoire des Alpes, 13, p. 83-98.

Benjamini Y., Hochberg Y. (1995) - Controlling the False Discovery Rate: a Practical and Powerful Approach to Multiple Testing, Journal of the Royal Statistical Society, Series $B$ (Methodological), 57, 1, p. 289-300.

Billard M. (2010) - Évolution des pathocénoses du Néolithique moyen à La Tène sur des séries ostéo-archéologiques de Limagne d'Auvergne (Puy-de-Dôme), in A. Beeching, É. Thirault et J. Vital (dir.), Économie et société à la fin de la Préhistoire : actualité de la recherche, actes des $7^{\text {es }}$ Rencontres méridionales de Préhistoire récente (Lyon-Bron, 3-4 novembre 2006), Lyon, ALPARA, p. 317-325.

BIRKNER R. (1980) - L'image radiologique typique du squelette, Paris, Maloine, $564 \mathrm{p}$.

Bocherens H., Fizet M., Mariotti A., Lange-Badre B., Vandermeersch B., Borel J.-P., Bellon G. (1991) - Isotopic Biogeochemistry $\left({ }^{13} \mathrm{C},{ }^{15} \mathrm{~N}\right)$ of Fossil Vertebrate Collagen: Application to the Study of Past Food Web Including Neanderthal Man, Journal of Human Evolution, 20, p. 481492.

Bocherens H. (1992) - Biogéochimie isotopique $\left({ }^{13} \mathrm{C},{ }^{15} \mathrm{~N}\right.$, $\left.{ }^{18} \mathrm{O}\right)$ et paléontologie des vertébrés : application à l'étude des réseaux trophiques révolus et des paléoenvironnements, thèse de doctorat, université Paris VI, 317 p.

Bocherens H. (1997) - Signature isotopique dans le collagène des os, Comptes rendus de la Société de biologie, 4, p. 493510 .

Bocherens H., Billiou D., Tresset A. (2005) - Approche biogéochimique $\left({ }^{13} \mathrm{C}\right.$ et $\left.{ }^{15} \mathrm{~N}\right)$ de l'exploitation de l'environnement par les humains, in F. Giligny (dir.), Louviers "La Vilette » (Eure) : un site Néolithique moyen en zone humide, Rennes, Presses universitaires de Rennes (Documents archéologiques), p. 265-268.
Bocherens H., Drucker D. (2003) - Trophic Level Isotopic Enrichment of Carbon and Nitrogen in Bone Collagen: Case Studies from Recent and Ancient Terrestrial Ecosystems, International Journal of Osteoarchaeology, 13, p. 46-53.

Bonsall C., Lennon R., Mcsweeney K., Stewart C., Harkness D., Boroneant V., Bartosiewicz L., Payton R., Chapman J. (1997) - Mesolithic and Early Neolithic in the Iron Gates: a Paleodietary Perspective, Journal of European Archaeology, 5, 1, p. 50-92.

Bösl C., Grupe G., Peters J. (2006) - A Late Neolithic Vertebrate Food Web Based on Stable Isotope Analyses, International Journal of Osteoarchaeology, 16, 4, p. 296-315.

Boulestin B. (2008) - Pourquoi mourir ensemble? À propos des tombes multiples dans le Néolithique français, Bulletin de la Société préhistorique française, 105, 1, p. 103-130.

BRÉHARD S. (2011) - Le complexe chasséen vu par l'archéozoologie : révision de la dichotomie Nord-Sud et confirmation de la partition fonctionnelle au sein des sites méridionaux, Bulletin de la Société préhistorique française, 108, 1, p. 1-20.

Bréhard S., Beeching A., Vigne J.-D. (2010) - Shepherds, Cowherds and Site Function on Middle Neolithic Sites of the Rhône Valley: An Archaeozoological Approach to the Organization of Territories and Societies, Journal of Anthropological Archaeology, 29, 2, p. 179-188.

Britton K., Müldner G., Bell M. (2008) - Stable Isotope Evidence for Salt-marsh Grazing in the Bronze Age Severn Estuary, UK: Implications for Palaeodietary Analysis at Coastal Sites, Journal of Archaeological Science, 35, 8, p. 2111-2118.

Brossier S., Marlière P. (2000) - Le site Chasséen de Villeneuve-Tolosane - Cugnaux, Haute-Garonne : une nouvelle opération de sauvetage (1996-1997), in M. Leduc, N. Valdeyron et J. Vaquer (dir.), Sociétés et espaces, actes des $3^{\text {es }}$ Rencontres méridionales de Préhistoire récente (Toulouse, 6-7 novembre 1998), Toulouse, Archives d'écologie préhistorique, p. 313-318.

Brugel C., Carmie H., Humbert L., Court E., Vrignaud S. (non publié) - Rapport pour la DREAL Auvergne. Annexes : poissons et invertébrés aquatiques. Approches par espèces, Clermont-Ferrand, office national de l'Eau et des Milieux aquatiques et DIREN Auvergne, p. 65.

Bruzek J. (2002) - A Method for Visual Determination of Sex, Using the Human Hip Bone, American Journal of Physical Anthropology, 117, p. 157-168.

Bruzek J., Schmitt A., Murail P. (2005) - Identification biologique individuelle en paléoanthropologie. Détermination du sexe et de l'âge au décès à partir du squelette, in O. Dutour, J.-J. Hublin et B. Vandermeersch (dir.), Objets et méthodes en Paléoanthropologie, Paris, CTHS, p. $217-$ 245.

Chambon P., Leclerc J. (2007) - Les tombes multiples dans le Néolithique français, Bulletin de la Société préhistorique française, 104, 2, p. 289-306. 
Civetta A., Schmitt A., Saliba-Serre B., Gisclon J.-L., Loison G. (2009) - Comparaison de deux «populations » de la deuxième moitié du $\mathrm{V}^{\mathrm{e}}$ millénaire avant notre ère : approche anthropométrique, Bulletins et mémoires de la Société d'anthropologie de Paris, 21, 3-4, p. 141-158.

Cleuvenot E., Houët F. (1993) - Proposition de nouvelles équations d'estimation de stature applicables pour un sexe indéterminé et basées sur les échantillons de Trotter et Gleser, Bulletins et mémoires de la Société d'anthropologie de Paris, 5, p. 245-255.

Deniro M. J. (1985) - Post-mortem Preservation and Alteration of In Vivo Bone Collagen Isotope Ratios on Relation to Palaeodietary Reconstruction, Nature, 317, 6032, p. 806809.

Deniro M. J., Epstein S. (1978) - Influence of Diet on the Distribution of Carbon Isotopes in Animals, Geochimica et Cosmochimica Acta, 42, p. 495-506.

Deniro M. J., Epstein S. (1981) - Influence of Diet on the Distribution of Nitrogen Isotopes in Animals, Geochimica et Cosmochimica Acta, 45, p. 341-351.

Drucker D. G., Bocherens H., Billiou D. (2003) - Evidence for Shifting Environmental Conditions in Southwestern France from 33,000 to 15,000 Years Ago Derived from Carbon-13 and Nitrogen-15 Natural Abundances in Collagen of Large Herbivores, Earth and Planetary Science Letters, 216, 1-2, p. 163-173.

Drucker D. G., Madelaine S., Morala A. (2011) - Les derniers rennes de Dordogne. Nouvelles données chronologiques et environnementales par l'étude isotopique du collagène $\left({ }^{13} \mathrm{C},{ }^{14} \mathrm{C}\right.$ et $\left.{ }^{15} \mathrm{~N}\right)$, Paleo, 22, p. 85-100.

Duday H., Vaquer J. (2003) - Les sépultures chasséennes $\mathrm{du}$ site des Plots à Berriac (Aude), in $\mathrm{P}$. Chambon et $\mathrm{J}$. Leclerc (dir.), Les pratiques funéraires néolithiques avant 3500 av. J.-C. en France et dans les régions limitrophes, actes de la table ronde de la SPF (Saint-Germain-en-Laye, 15-17 juin 2001), Paris, Société préhistorique française (Mémoire, 33), p. 73-80.

Dürrwächter C., Craig O. E., Collins M., Burger J., Alt K. W. (2006) - Beyond the Grave: Variability in Neolithic Diets in Southern Germany?, Journal of Archaeological Science, 33, p. 39-48.

Fogel M. L., Tuross N., Owsley D. W. (1989) - Nitrogen Isotope Tracers of Human Lactation in Modern and Archaeological Populations, Annual Report of Geophysical Laboratory Carnegie Institution of Washington, p. 111-117.

GANDELIN M. (2011) - Les enceintes chasséennes de Villeneuve-Tolosane et de Cugnaux dans leur contexte du Néolithique moyen européen, Toulouse, Archives d'écologie préhistorique, $506 \mathrm{p}$

Gandelin M., Vaquer J. (2008) - Présentations des sites chasséens de Villeneuve-Tolosane et de Cugnaux et localisation des faits funéraires, in J. Vaquer, M. Gandelin, M. Remicourt et Y. Tcheremissinoff (dir.), Défunts néolithiques en Toulousain, Toulouse, Archives d'écologie préhistorique, p. 29-40.

GARINE I. DE (2007) - À propos de l'alimentation en milieu montagnard, in G. Boëtsch et A. Hubert (dir.), L'alimenta- tion en montagne, Gap, Éditions des Hautes-Alpes, p. 313317.

Goude G. (2007) - Étude des modes de subsistance de populations néolithiques (VI $I^{e}-I V^{e}$ millénaires av. J.-C.) dans le Nord-Ouest de la Méditerranée. Approche par l'utilisation des isotopes stables $\left({ }^{13} \mathrm{Cet}^{15} \mathrm{~N}\right) \mathrm{du}$ collagène, thèse de doctorat, université Bordeaux 1 et université de Leipzig, 415 p.

Herrscher E. (2003) - Alimentation d'une population historique : analyse des données isotopiques de la nécropole de Saint-Laurent-de-Grenoble (XIII ${ }^{\mathrm{e}}-\mathrm{XV}^{\mathrm{e}}$ siècle, France), Bulletins et mémoires de la Société d'anthropologie de Paris, 15, 3-4, p. 149-269.

Herrscher E. (2011) - Inferring Diet by Stable Isotope Analysis: a Case Study from the French Alps, in M. Carver (dir.), The Archaeology of Medieval Europe, 2. The Twelfth to Sixteenth Centuries AD, Aarhus, Aarhus University Press, p. 139-146.

Herrscher E. (2013) - Détection isotopique des modalités d'allaitement et de sevrage à partir des ossements archéologiques, Cahiers de nutrition et de diététique, 48 [en ligne].

Herrscher E., Bocherens H., Valentin F., Colardelle R. (2001) - Comportement alimentaire au Moyen Âge à Grenoble : application de la biogéochimie isotopique à la nécropole de Saint-Laurent (XIII ${ }^{\mathrm{e}}-\mathrm{XV}^{\mathrm{e}}$ siècles, Isère, France), Comptes rendus de l'Académie des sciences. Série 2, Sciences de la Vie, 324, p. 479-487.

Herrscher E., Valentin F., Bocherens H., Colardelle R. (2007) - Les squelettes de Saint-Laurent-de-Grenoble, des témoins de l'alimentation et de la santé au Moyen Âge (XIII ${ }^{\mathrm{e}}-\mathrm{XV}^{\mathrm{e}}$ siècles, France), in F. Audoin-Rouzeau et F. Sabban (dir.), Un aliment sain dans un corps sain. Perspectives historiques, Tours, Presses universitaires François-Rabelais, p. 123-138.

Herrscher E., Le Bras-Goude G. (2008) - Viande, laitage, poissons ou végétaux. Approche biochimique de l'alimentation néolithique en Toulousain, in J. Vaquer, M. Gandelin, M. Remicourt et Y. Tchérémissinoff (dir.), Défunts néolithiques en Toulousain, Toulouse, Archives d'écologie préhistorique, p. 199-208.

Herrscher E., Hervieu P., Bailly-Maitre M.-C., MarTIN L. (2009) - Subsistance d'une population minière en milieu extrême au Moyen Âge (Brandes-en-Oisans, Isère) : apport des données ostéologiques et isotopiques, Bulletins et mémoires de la Société d'anthropologie de Paris, 20, 3-4, p. 238.

Herrscher E., Le Bras-Goude G. (2010) - Southern French Neolithic Populations: Isotopic Evidence for Regional Specificities in Environment and Diet, American Journal of Physical Anthropology, 14, 2, p. 259-272.

Herrscher E., Thomas A., Chambon P., Mordant D. (2012) - Pratiques alimentaires et nécropoles monumentales au Néolithique : le cas des sujets du site de Balloy-les-Raudins (culture de Cerny, Ve millénaire, Bassin parisien), Bulletins et mémoires de la Société d'anthropologie de Paris, 23, S21.

Katzenberg M. A. (1993) - Age Differences and Population Variation in Stable Isotope Values from Ontario Canada, in J.-B. Lambert et G. Grupe (dir.), Prehistoric Human Bone Archaeology at the Molecular Level, Berlin, Springer, p. 39-62. 
Le Bras-Goude G. (2008) - Diet of Ancient and Middle Neolithic Populations in the Northwest of Mediterranean. Anthropological and Isotopic Studies, in L. Nuninger, F. Favory et C. Mordant (dir.), Settlement Pattern, Production and Trades from Neolithic to Middle Ages. Archaedyn: Seven Millennia of Territorial Dynamics, actes du symposium international (Dijon, 22-25 juin), Oxford, Archaeopress (British Archaeological Reports, International Series 2370), p. 133-138.

Le Bras-Goude G., Claustre F. (2009) - Exploitation of Domestic Mammals in the Eastern Pyrenees During the Neolithic Human Dietary Patterns at the Site of Montou (Corberes-les-Cabanes, France) Using Bone Collagen Stable Isotopes $\left(\delta^{13} \mathrm{C}, \delta^{15} \mathrm{~N}\right)$, Vie et Milieu - Life and Environment, 59, 2, p. 219-225.

Le Bras-Goude G., Herrscher E., Vaquer J. (sous presse) - Funeral Practices and Foodstuff Behavior: What Does Eat Meat Mean? Stable Isotope Analysis of Middle Neolithic Populations in Languedoc Region (France), Journal of Anthropological Archaeology.

Le Bras-Goude G., Lisfranc R., Loison G., Forest V., Herrscher E. (2011) - The Bronze Age Diet in Auvergne (France): a Stable Isotope Approach, American Journal of Physical Anthropology, 144, Issue Supplement 52, p. 195196.

Le Bras-Goude G., Schmitt A., Loison G. (2009) - Comportements alimentaires, aspects biologiques et sociaux au Néolithique : le cas du Crès (Hérault, France), Comptes rendus Palevol, 8, p. 79-91.

Lidén K., Eriksson G., Nordqvist B., Götherström A., Bendixen E. (2004) - The Wet and the Wild Followed by the Dry and the Tame, or Did They Occur at the Same Time? Diet in Mesolithic and Neolithic Southern Sweden, Antiquity, 78, 299, p. 23-33.

Lillie M. C., Richards M. (2000) - Stable Isotope Analysis and Dental Evidence of Diet at the Mesolithic-Neolithic Transition in Ukraine, Journal of Archaeological Science, 27, p. 965-972.

Loison G. (1998) - La nécropole de Pontcharaud en basse Auvergne, in J. Guilaine (dir.), Sépultures d'Occident et genèses des mégalithismes, Paris, Errance, p. 189-206.

Loison G., Fabre V., Villemeur I. (2004) - Le Crès. Habitats préhistoriques en bordure de l'Orb. Structures domestiques et sépulture du Chasséen ancien, rapport final d'opération, Montpellier, INRAP, $304 \mathrm{p}$.

Loison G., Gandelin M., Vergély H., Gleize Y., Tchérémissinoff Y., Haurillon R., Marsac R., Remicourt M., Torchy L., Vinolas F. (2011) - Dynamiques d'occupation des sols à la Préhistoire récente dans la basse vallée de l'Hérault : les apports de l'A75, tronçon PézenasBéziers, in I. Sénépart, T. Perrin, E. Thirault et S. Bonnardin (dir.), Marges, frontières et transgressions : actualité de la recherche, actes des $8^{\text {es }}$ Rencontres méridionales de Préhistoire récente (Marseille, 7-8 nov 2008), Toulouse, Archives d'écologie préhistorique, p. 317-344.

Loison G., Sснмiтt A. (2009) - Diversité des pratiques funéraires et espaces sépulcraux sectorisés au Chasséen ancien sur le site du Crès à Beziers (Hérault), Gallia Préhistoire, 51, p. 245-272.
Longin R. (1971) - New Method of Collagen Extraction for Radiocarbon Dating, Nature, 230, p. 241-242.

Lubell D., Jackes M., Schwarcz H., Knyf M., MeikleJOHN C. (1994) - The Mesolithic-Neolithic Transition in Portugal: Isotopic and Dental Evidence of Diet, Journal of Archaeological Science, 21, p. 201-216.

Mafart B. (1989) - Intérêt et limite de l'étude de quelques marqueurs osseux de la malnutrition au cours de la croissance, in L. Buchet (dir.), Homme et milieu : approches paléoanthropologiques, actes des $4^{\text {es }}$ Journées anthropologiques de Valbonne (Valbonne, 25-27 mai 1988), Paris, CNRS, p. 73-84.

Milner N., Craig O. E., Bailey G. N., Pedersen K., Andersen S. H. (2004) - Something Fishy in the Neolithic? A Re-evaluation of Stable Isotope Analysis of Mesolithic and Neolithic Coastal Populations, Antiquity, 78, 299, p. 9-22.

Minagawa M., Wada E. (1984) - Stepwise Enrichment of ${ }^{15} \mathrm{~N}$ Along Food Chain: Further Evidence and the Relation between $\delta^{15} \mathrm{~N}$ and Animal Age, Geochimica et Cosmochimica Acta, 48, 5, p. 1135-1140.

Murail P., Bruzek J., Houët F., Cunha E. (2005) - DSP: a Tool for Probabilistic Sex Diagnosis Using Worldwide Variability in Hip-bone Measurement, Bulletins et mémoires de la Société d'anthropologie de Paris, 17, p. 167-176.

Olivier G., Pineau H. (1958) - Détermination de l'âge du fœtus et de l'embryon, Archives d'anatomie (La semaine des hôpitaux), 6, p. 21-28.

PARK R. B., EPSTEIN S. (1960) - Carbon Isotope Fractionnation during Photosynthesis, Geochimica et Cosmochimica Acta, 21, p. 110-126.

Polet C., Orban R. (2001) - Dents et ossements humains : que mangeait-on au Moyen Âge?, Turhnout, Brepols, 173 p.

R Development Core Team (2011) - R: A Language and Environment for Statistical Computing, Vienne (Autriche), R Foundation for Statistical Computing, $409 \mathrm{p}$.

RICHARDS M. (2003) - Explaining the Dietary Isotope Evidence for the Rapid Adoption of the Neolithic in Britain, in M. Parker Pearson (dir.), Food Culture and Identity in the Neolithic and Early Bronze Age, Oxford, Archaeopress (British Archaeological Reports, International Series 1117), p. 31-36.

Rodière É., Bocherens H., Angibault J.-M., Mariotti A. (1996) - Particularités isotopiques de l'azote chez le chevreuil (Capreolus capreolus L.) : implications pour les reconstitutions paléoenvironnementales, Comptes rendus de l'Académie des sciences. Série 3, Sciences de la vie, 323, p. 179-185.

Saliba-Serre B., Schmitt A. (2012) - Difficultés liées à l'application de la diagnose sexuelle secondaire dans la série néolithique de Poncharaud II (Puy-de-Dôme, France), in résumés de la $1837^{\circ}$ réunion scientifique de la Société d'anthropologie de Paris (Bordeaux, 25-27 janvier 2012), Bulletins et mémoires de la Société d'anthropologie de Paris, 20, p. 23.

Scheuer L., Black S. (2000) - Developmental Juvenile Osteology, Londres, Academic Press, 587 p. 
Schmitr A. (2005) - Une nouvelle méthode pour estimer l'âge au décès des adultes à partir de la surface sacro-pelvienne iliaque, Bulletins et mémoires de la Société d'anthropologie de Paris, 17, 1-2, p. 89-101.

Schмiтt A. (2008) - Une nouvelle méthode pour estimer l'âge des individus décédés avant et après 40 ans, Journal de médecine légale et de droit médical, 51, 1, p. 17-24.

Schmitt A., Georges P. (2008) - Quelle démarche suivre pour estimer l'âge au décès à partir du squelette? in $\mathrm{P}$. Charlier (dir.), Manuel pratique de paléopathologie humaine, Paris, De Boccard, p. 269-280

Schoeninger M. J., De Niro M. J., Tauber H. (1983) - Stable Nitrogen Isotope Ratios of Bone Collagen Reflect Marine and Terrestrial Components of Prehistoric Human Diet, Science, 220, p. 1381-1383.

Schoeninger M. J., Moore K. (1992) - Bone Stable Isotope Studies in Archaeology, Journal of World Prehistory, 6, 2, p. $247-285$.

SCHURR M. R. (1998) - Using Stable Nitrogen Isotope to Study Weaning Behavior in Past Populations, World Archaeology, 30, 2, p. 327-342.

Soto-Heim P. (2001) - Considérations sur la stature des populations sub-actuelles de Patagonie et extrême Sud d'Amérique, Biométrie humaine et anthropologie, 19, 3-4, p. 257264.

Stuiver M., Reimer P. J. (1993) - Extended ${ }^{14} \mathrm{C}$ Data Base and Revised CALIB 3.0 ${ }^{14} \mathrm{C}$ Age Calibration Programme, Radiocarbon, 35, p. 215-230.

Susanne C. (1993) - Croissance et nutrition, Bulletins et mémoires de la Société d'anthropologie de Paris, 5, 1-2, p. 69-83.

Tauber H. (1981) $-{ }^{13} \mathrm{C}$ Evidence for Dietary Habits of Prehistoric Man in Denmark, Nature, 292, p. 332-333.

TCHÉRÉMISSINOFF Y. (2003) - Les sépultures chasséennes de Narbons (Haute Garonne) : description comparaison et fonctionnement, in P. Chambon et J. Leclerc (dir.), Les pratiques funéraires néolithiques avant $3500 \mathrm{av}$. J.-C. en France et dans les régions limitrophes, actes de la table ronde de la SPF (Saint-Germain-en-Laye, 15-17 juin 2001), Paris, Société préhistorique française (Mémoire, 33), p. 81-90.

Tchérémissinoff Y., Martin H., Texier M., Vaquer J. (2005) - Les sépultures chasséennes du site de Narbons à Montesquieu-de-Lauragais (Haute-Garonne), Gallia Préhistoire, 47, p. 1-32.

Testart A. (2004) - Les morts d'accompagnement : la servitude volontaire I, Paris, Errance, 261 p.

Trément F., Loison G., Argant J., Bréhéret J.-G., CabAnis M., Dousteyssier B., Fourmont A., Fournier G., Liabeuf R., Lopez-Saez J.-A. (2006) - Interactions sociétés-milieux en Grande Limagne du Néolithique à l'époque romaine. Apport des recherches interdisciplinaires conduites dans le bassin de Sarliève (Puy-de-Dôme), in P. Fouéré, C. Chevillot et P. Courtaud (dir.), Paysages et peuplements : aspects culturels et chronologie en France méridionale, actes des $6^{\text {es }}$ Rencontres méridionales de Préhistoire récente (Périgueux, 14-16 octobre 2004), Périgueux, ADRAHP-PS, p. 11-32.

Ubelaker D. H. (1989) - The Estimation of Age at Death from Immature Human Bone, in M. Y. Iscan (dir.), Age Markers in the Human Skeleton, Springfield (Ill.), Charles C. Thomas, p. 55-70.

UliJasnek S. J. (2003) - Trends in Body Size, Diet and Food Avilability in the Cook Islands in the Second Half of the 20th Century, Economics and Human Biology, 1, 1, p. 123127.

Valentin J. (2003) - Basic Anatomical and Physiological Data for Use in Radiological Protection: Reference Values, Oxford, Pergamon Press (ICRP Publication, 89), 280 p.

Van der Merwe N. J., Medina E. (1991) - The Canopy Effect Carbon Isotope Ratios and Foodwebs in Amazonia, Journal of Archaeological Science, 18, p. 249-259.

VAN Klinken G. J. (1999) - Bone Collagen Quality Indicators for Palaeodietary and Radiocarbon Measurements, Journal of Archaeological Science, 26, p. 687-695.

\section{Gwenaëlle Goude Estelle HERrSCHER, Sandrine CABUT Guy ANDRÉ}

UMR 7269 « LAMPEA »

MMSH, 5 rue du Château-de-l'Horloge BP 647, 13094 Aix-en-Provence cedex 2 goude@mmsh.univ-aix.fr

Aurore SCHMITT UMR 7268 «ADES » Université Aix-Marseille Faculté de médecine nord Bd Pierre-Dramard, bât A, CS 80011 13344 Marseille cedex 15

Gilles LoIson INRAP Méditerranée 561, rue Étienne-Lenoir, Km Delta 30900 Nîmes 